\title{
A SHARP QUANTITATIVE VERSION OF ALEXANDROV'S THEOREM VIA THE METHOD OF MOVING PLANES
}

\author{
GIULIO CIRAOLO, LUIGI VEZZONI
}

\begin{abstract}
We prove the following quantitative version of the celebrated Soap Bubble Theorem of Alexandrov. Let $S$ be a $C^{2}$ closed embedded hypersurface of $\mathbb{R}^{n+1}, n \geq 1$, and denote by $\operatorname{osc}(H)$ the oscillation of its mean curvature. We prove that there exists a positive $\varepsilon$, depending on $n$ and upper bounds on the area and the $C^{2}$-regularity of $S$, such that if $\operatorname{osc}(H) \leq \varepsilon$ then there exist two concentric balls $B_{r_{i}}$ and $B_{r_{e}}$ such that $S \subset \bar{B}_{r_{e}} \backslash B_{r_{i}}$ and $r_{e}-r_{i} \leq C \operatorname{osc}(H)$, with $C$ depending only on $n$ and upper bounds on the surface area of $S$ and the $C^{2}$ regularity of $S$. Our approach is based on a quantitative study of the method of moving planes and the quantitative estimate on $r_{e}-r_{i}$ we obtain is optimal.

As a consequence of this theorem, we also prove that if $\operatorname{osc}(H)$ is small then $S$ is diffeomorphic to a sphere and give a quantitative bound which implies that $S$ is $C^{1}$-close to a sphere.
\end{abstract}

\section{Contents}

1. Introduction

2. Notation and preliminary results

2.1. Classical solutions to mean curvature equation 7

2.2. The symmetry result of Alexandrov 11

2.3. Curvatures of projected surfaces

3. Technical Lemmas 15

4. Proof of Theorem 1.1 20

4.1. Step 1. Approximate symmetry in one direction 20

4.2. Steps 2-3. Approximate radial symmetry and conclusion 29

5. Proof of Corollary 1.2

References 32

\section{INTRODUCTION}

The Soap Bubble Theorem proved by Alexandrov in [3] has been the object of many investigations. In its simplest form it states that

The $n$-dimensional sphere is the only compact connected embedded hypersurface of $\mathbb{R}^{n+1}$ with constant mean curvature.

As it is well-known, the embeddedness condition is necessary, as implied by the celebrated counterexamples by Hsiang-Teng-Yu [28] and Wente [48]. There have been several extensions of the rigidity result of Alexandrov to more general settings. Alexandrov proved this Theorem

Date: January 13, 2016.

1991 Mathematics Subject Classification. Primary 35B50, 35B51; Secondary 53C20, 53C21.

Key words and phrases. Alexandrov Soap Bubble Theorem, method of moving planes, stability, mean curvature, pinching. 
in a more general setting; in particular, the Euclidean space can be replaced by any space of constant curvature (see also [4] where he discussed several possible generalizations). Montiel and Ros [41] and Korevaar [30] studied the case of hypersurfaces with constant higher order mean curvatures embedded in space forms. Alexandrov Theorem has been studied also for warped product manifolds by Montiel [39], Brendle [10] and Brendle and Eichmair [11. There are many other related results; the interested reader can refer to [12, 16, 15, 24, 29, 42, 43, 44, 49] and references therein.

To prove the Soap Bubble Theorem, Alexandrov introduced the method of moving planes, a very powerful technique which has been the source of many insights in analysis and differential geometry. Serrin understood that the method can be applied to Partial Differential Equations. Indeed, in his seminal paper [45] he obtained a symmetry result for the torsion problem which gave rise to a huge amount of results for overdetermined problems (the interest reader can refer to the references in [18]). In [25] Gidas, Ni and Nirenberg refined Serrin's argument to obtain several symmetry results for positive solutions of second order elliptic equations in bounded and unbounded domains (see also [36] and [37]). The method was further employed by Caffarelli, Gidas and Spruck [13] to prove asymptotic radial symmetry of positive solutions for the conformal scalar curvature equation and others semilinear elliptic equations (see also [34]). The moving planes were also used to obtain several celebrated results in differential geometry: Schoen [47] characterized the catenoid, Meeks [38] and Korevaar, Kusner and Solomon [33] showed that a complete connected properly embedded constant mean curvature surface in the Euclidean space with two annuli ends is rotationally symmetric. There is a large amount of other interesting papers on these topics which are not mentioned here.

Alexandrov's proof in the Euclidean space works as follows: (i) show that for any direction $\omega$ there exists a critical hyperplane orthogonal to $\omega$ which is of symmetry for the surface $S$; (ii) since the center of mass $\mathcal{O}$ of $S$ lies on each hyperplane of symmetry, then every hyperplane passing through $\mathcal{O}$ is of reflection symmetry for $S$; (iii) since any rotation about $\mathcal{O}$ can be written as a composition of $n+1$ reflections, then $S$ is rotationally invariant, which implies that $S$ is the $n$-dimensional sphere. The crucial step in this proof is (i), which is obtained by applying the method of moving planes and using maximum principle (see Theorem A in Subsection 2.2).

In this paper we study a quantitative version of the Soap Bubble Theorem, that is we assume that the oscillation of the mean curvature $\operatorname{osc}(H)$ is small and we prove that $S$ is close to a sphere. More precisely, let $S$ be an $n$-dimensional, $C^{2}$-regular, connected, closed hypersurface embedded in $\mathbb{R}^{n+1}$, and denote by $|S|$ the area of $S$. Since $S$ is $C^{2}$ regular, then it satisfies a uniform touching sphere condition of (optimal) radius $\rho$. We orientate $S$ according to the inner normal. Given $p \in S$, we denote by $H(p)$ the mean curvature of $S$ at $p$, and we let

$$
\operatorname{osc}(H)=\max _{p \in S} H(p)-\min _{p \in S} H(p) .
$$

Our main result is the following theorem.

Theorem 1.1. Let $S$ be an $n$-dimensional, $C^{2}$-regular, connected, closed hypersurface embedded in $\mathbb{R}^{n+1}$. There exist constants $\varepsilon, C>0$ such that if

$$
\operatorname{osc}(H) \leq \varepsilon
$$

then there are two concentric balls $B_{r_{i}}$ and $B_{r_{e}}$ such that

$$
S \subset \bar{B}_{r_{e}} \backslash B_{r_{i}}
$$

and

$$
r_{e}-r_{i} \leq C \operatorname{osc}(H) .
$$


The constants $\varepsilon$ and $C$ depend only on $n$ and upper bounds on $\rho^{-1}$ and $|S|$.

Under the assumption that $S$ bounds a convex domain, there exist some results in the spirit of Theorem 1.1 which are available in literature. In particular, when the domain is an ovaloid, the problem was studied by Koutroufiotis [32, Lang [35] and Moore [40]. Other stability results can be found in Schneider [47] and Arnold [7]. These results were improved by Kohlmann in [31] where he proved an explicit Hölder type stability in (3). In Theorem 1.1, we do not consider any convexity assumption and we obtain the optimal rate of stability in (3), as can be proven by a simple calculation for ellipsoids.

Theorem 1.1 has a quite interesting consequence which we explain in the following. It is well-known (see for instance [27]) that if every principal curvature $\kappa_{i}$ of $S$ is pinched between two positive numbers, i.e.

$$
\frac{1}{r} \leq \kappa_{i} \leq(1+\delta) \frac{1}{r}, \quad i=1, \ldots, n,
$$

then $S$ is close to a sphere of radius $r$. Following Gromov [27, Remark (c), p.67-68], one can ask what happens when only the mean curvature is pinched. We have the following result.

Corollary 1.2. Let $\rho_{0}, A_{0}>0$ and $n \in \mathbb{N}$ be fixed. There exists a positive constant $\varepsilon$, depending on $n, \rho_{0}$ and $A_{0}$, such that if $S$ is a connected closed $C^{2}$ hypersurface embedded in $\mathbb{R}^{n+1}$ with $|S| \leq A_{0}, \rho \geq \rho_{0}$, whose mean curvature $H$ satisfies

$$
\operatorname{osc}(H)<\varepsilon,
$$

then $S$ is diffeomorphic to a sphere.

Moreover $S$ is $C^{1}$-close to a sphere, i.e. there exists a $C^{1}$-map $F=I d+\Psi \nu: \partial B_{r_{i}} \rightarrow S$ such that

$$
\|\Psi\|_{C^{1}\left(\partial B_{r_{i}}\right)} \leq C(\operatorname{osc}(H))^{\frac{1}{2}}
$$

where $C$ depends only on $n$ and upper bounds on $\rho^{-1}$ and $|S|$.

Before explaining the argument of the proof of Theorem 1.1, we give a couple of remarks on the bounds on $\rho$ and $|S|$ in Theorem 1.1 and its Corollary 1.2. The upper bound on $\rho^{-1}$ controls the $C^{2}$ regularity of the hypersurface, which is a crucial condition for obtaining an estimate like (3). Indeed, if we assume that $\rho$ is not bounded from below, it is possible to construct a family of closed surfaces embedded in $\mathbb{R}^{3}$, not diffeomorphic to a sphere, with osc $(H)$ arbitrarly small and such that (3) fails (see Remark 5.2 and [17]). The upper bound on $|S|$ is a control on the constants $\varepsilon$ and $C$, which clearly change under dilatations.

We remark that Corollary 1.2 can be obtained by a compactness argument by using the theory of varifolds by Allard [5] and Almgren [6]. Indeed, by Allard's compactness theorem every sequence of closed hypersurfaces satisfying (uniformly) the assumptions of Corollary 1.2 admits a subsequence which, up to translations, converges to a hypersurface which satisfies a touching ball condition and hence is $C^{1,1}$ regular. By standard regularity theory, the hypersurface is smooth and is a sphere by the classical Alexandrov theorem. We think that also the stability estimates in Theorem 1.1 can be obtained by using Allard's regularity theorem.

There are other possible strategies to obtain quantitative estimates for almost constant mean curvature hypersurfaces and give results as in the spirit of Theorem 1.1. Indeed, as we already mentioned, there are several proofs of the rigidity result of Alexandrov (i.e. when $H$ is constant). Beside the method of moving planes (which will be our approach), one could try to quantitavely study the proofs in [41, [42] and [44], which are based on integral identities. For instance, the approach in [17] starts form [44] and finds quantitative estimates on the closedness of the hypersurface to a compound of tangent balls. As explained in [17, Appendix A], another possible 
approach would be to start from the proof in [41] and then study almost umbilical hypersurfaces, in the same spirit as 22 and 23 . However, these approaches based on integral identities do not seem to lead to optimal estimates as in our Theorem 1.1 (see [17] for a detailed discussion).

Our approach, instead, is based on a quantitative analysis of the method of moving planes and uses arguments from elliptic PDEs theory. Since the proof of symmetry is based on maximum principle, our proof of the stability result will make use of Harnack's and Carleson's (or boundary Harnack's) inequalities and the Hopf Lemma, which can be considered as the quantitative counterpart of the strong and boundary maximum principles. We emphasize that the stability estimate (3) is optimal and that our proof permits to compute the constants explicitly.

A quantitative study of the method of moving planes was first performed in [1], where the authors obtained a stability result for Serrin's overdetermined problem [45, and it has been used in a series of paper by the first author [19, 20, 21] for studying the stability of radial symmetry for Serrin's and other overdetermined problems (see also [9] for an approach based on integral identities). In this paper, we follow the same approach of [1], but the setting here is complicated by the fact that we have to deal with manifolds. As we will show, the main goal is to prove an approximate symmetry result for one (arbitrary) direction. After that, the approximate radial symmetry is well-established and follows by an argument in [1]. To prove the approximate symmetry in one direction, we apply the method of moving planes and show that the union of the maximal cap and of its reflection provides a set that fits well $S$. This is the main point of our paper and is achieved by developing the following argument. Assume that the surface and the reflected cap are tangent at some point $p_{0}$ which is an interior point of the reflected cap, and write the two surfaces as graphs of function in a neighborhood of $p_{0}$. The difference $w$ of these two functions satisfies an elliptic equation $L w=f$, where $\|f\|_{\infty}$ is bounded by $\operatorname{osc}(H)$. By applying Harnack's inequality and interior regularity estimates, we have a bound on the $C^{1}$ norm of $w$, which says that the two graphs are close in $C^{1}$ norm no more than some constant times $\operatorname{osc}(H)$. It is important to observe that this estimate implies that the two surfaces are close to each other and also that the two corresponding Gauss maps are close (in some sense) in that neighborhood of $p_{0}$. Then we connect any point $p$ of the reflected cap to $p_{0}$ and we show that such closeness propagates at $p$. Since we are dealing with a manifold, we have to change local parameterization while we are moving from $p_{0}$ to $p$ and we have to prove that the closeness information is preserved. By using careful estimates and making use of interior and boundary Harnack's inequalities, we show that this is possible if we assume that $\operatorname{osc}(H)$ is smaller than some fixed constant.

The paper is organized as follows. In Section 2 we prove some preliminary results about hypersurfaces in $\mathbb{R}^{n+1}$, we recall some results on classical solutions to mean curvature type equations, and we give a sketch of the proof of the symmetry result of Alexandrov. In Section 3 we prove some technical lemmas which will be used for proving the stability result. In Sections 4 and 5 we prove Theorem 1.1 and Corollary 1.2 respectively.

Acknowledgements. The authors wish to thank Manuel Del Pino, Francesco Maggi, Antonio Ros and Susanna Terracini for their enlightening remarks and useful discussions we had together. The problem faced in the paper was suggested to the first author by Rolando Magnanini, who is also acknowledged for his interest in the work. Morerover, the authors would like to thank also Bang-Yen Chen, Barbara Nelli, Paolo Piccione, Fabio Podestà and Paolo Salani for useful conversations. We also thank the anonymous referees for their remarks and for suggesting an improvement of Corollary 1.2 .

The paper was completed while the first author was visiting "The Institute for Computational Engineering and Sciences"(ICES) of The University of Texas at Austin, and he wishes to thank 
the institute for hospitality and support. The first author has been supported by the NSFDMS Grant 1361122, the "Gruppo Nazionale per l'Analisi Matematica, la Probabilità e le loro Applicazioni" (GNAMPA) of the Istituto Nazionale di Alta Matematica (INdAM) and the project FIRB 2013 "Geometrical and Qualitative aspects of PDE".

The second author was supported by the project FIRB "Geometria differenziale e teoria geometrica delle funzioni", the project PRIN "Varietà reali e complesse: geometria, topologia e analisi armonica" and by GNSAGA of INdAM.

\section{NotATION AND PRELIMINARY RESUlts}

In this section we collect some preliminary results which will be useful in the following. Although some of them are already known, we sketch their proofs for sake of completeness and in order to explain the notation which it will be adopted in the sequel.

Let $S$ be a $C^{2}$ regular, connected, closed hypersurface embedded in $\mathbb{R}^{n+1}, n \geq 1$, and let $\Omega$ be the relatively compact domain of $\mathbb{R}^{n+1}$ bounded by $S$. We denote by $T_{p} S$ the tangent hyperplane to $S$ at $p$ and by $\nu_{p}$ the inward normal vector. Given a point $\xi \in \mathbb{R}^{n+1}$ and an $r>0$, we denote by $B_{r}(\xi)$ the ball in $\mathbb{R}^{n+1}$ of radius $r$ centered at $\xi$. When a ball is centered at the origin $O$, we simply write $B_{r}$ instead of $B_{r}(O)$.

Let $\operatorname{dist}_{S}: \mathbb{R}^{n+1} \rightarrow \mathbb{R}$ be the distance function from $S$, i.e.

$$
\operatorname{dist}_{S}(\xi)= \begin{cases}\operatorname{dist}(\xi, S), & \text { if } \xi \in \Omega, \\ -\operatorname{dist}(\xi, S), & \text { if } \xi \in \mathbb{R}^{n+1} \backslash \Omega ;\end{cases}
$$

it is clear that $S=\left\{\xi \in \mathbb{R}^{n+1}: \operatorname{dist}_{S}(\xi)=0\right\}$. Moreover, it is well-known (see e.g. [26]) that $\operatorname{dist}_{S}$ is Lipschitz continuous with Lipschitz constant 1 and that it is of class $C^{2}$ in an open neighborhood of $S$. Therefore the implicit function theorem implies that, given a point $p \in S$, $S$ can be locally represented as a graph over the tangent hyperplane $T_{p} S$ : there exist an open neighbourhood $\mathcal{U}_{r}(p)$ of $p$ in $S$ and a $C^{2}$-map $u: B_{r} \cap T_{p} S \rightarrow \mathbb{R}$ such that

$$
\mathcal{U}_{r}(p)=\left\{p+x+u(x) \nu_{p}: x \in B_{r} \cap T_{p} S\right\} .
$$

Moreover, if $q=p+x+u(x) \nu_{p}$, with $x \in B_{r}(p) \cap T_{p} S$, we have

$$
\nu_{q}=\frac{\nu_{p}-\nabla u(x)}{\sqrt{1+|\nabla u(x)|^{2}}}
$$

where

$$
\nabla u(x)=\sum_{i=1}^{N} \partial_{e_{i}} u(x) e_{i},
$$

and $\left\{e_{1}, \ldots, e_{n}\right\}$ is an arbitrary orthonormal basis of $T_{p} S$. We notice that, according with the definition above, $\nabla u(x)$ is a vector in $\mathbb{R}^{n+1}$ for every $x$ in the domain of $u$. Moreover $\nu_{q} \cdot \nu_{p}>0$ for every $q \in B_{r} \cap T_{p} S$ and, if $|\nabla u|$ is uniformly bounded in $B_{r} \cap T_{p} S$, then $u$ can be extended to $B_{r^{\prime}} \cap T_{p} S$ with $r^{\prime}>r$.

Since $S$ is $C^{2}$-regular, then the domain $\Omega$ satisfies a uniform touching ball condition and we denote by $\rho$ the optimal radius, that is: for any $p \in S$ there exist two balls of radius $\rho$ centered at $c^{-} \in \Omega$ and $c^{+} \in \mathbb{R}^{n+1} \backslash \bar{\Omega}$ such that $B_{\rho}\left(c^{-}\right) \subset \Omega, B_{\rho}\left(c^{+}\right) \subset \mathbb{R}^{n+1} \backslash \bar{\Omega}$, and $p \in \partial B_{\rho}\left(c^{ \pm}\right)$. $B_{\rho}\left(c^{-}\right)$and $B_{\rho}\left(c^{+}\right)$are called, respectively, the interior and exterior touching balls at $p$.

In the following Lemma we show that we may assume $r=\rho$ in the definition of (5), and we give some bounds in terms of $\rho$ which will be useful in the sequel. 
Lemma 2.1. Let $p \in S$. There exists a $C^{2}$ map $u: B_{\rho} \cap T_{p} S \rightarrow \mathbb{R}$ such that

$$
\mathcal{U}_{\rho}(p)=\left\{p+x+u(x) \nu_{p}: x \in B_{\rho} \cap T_{p} S\right\}
$$

is a relative open set of $S$ and

$$
\begin{aligned}
& |u(x)| \leq \rho-\sqrt{\rho^{2}-|x|^{2}}, \\
& |\nabla u(x)| \leq \frac{|x|}{\sqrt{\rho^{2}-|x|^{2}}},
\end{aligned}
$$

for every $x \in B_{\rho} \cap T_{p} S$. Moreover

$$
\nu_{p} \cdot \nu_{q} \geq \frac{1}{\rho} \sqrt{\rho^{2}-|x|^{2}}, \quad \text { and } \quad\left|\nu_{p}-\nu_{q}\right| \leq \sqrt{2} \frac{|x|}{\rho},
$$

for every $q=p+x+u(x) \nu_{p}$ in $\mathcal{U}_{\rho}(p)$.

Proof. By the implicit function theorem, there exists $r>0, u: B_{r} \cap T_{p} S \rightarrow \mathbb{R}$ and $\mathcal{U}_{r}(p)$ as in (5). We may assume that $r \leq \rho$. The bound (7) in $B_{r} \cap T_{p} S$ easily follows from the definition of interior and exterior touching balls at $p$. We prove that estimate (8) in $B_{r} \cap T_{p} S$, which allows us to enlarge the domain of $u$ up to $B_{\rho} \cap T_{p} S$. Let

$$
q=p+x+u(x) \nu_{p}
$$

with $|x|<r$ be an arbitrary point of $\mathcal{U}_{r}(p)$ (notice that $\nu_{p} \cdot \nu_{q}>0$ ). Since

$$
B_{\rho}\left(p+\rho \nu_{p}\right) \cap B_{\rho}\left(q-\rho \nu_{q}\right)=\emptyset,
$$

we have that

$$
\left|p+\rho \nu_{p}-q+\rho \nu_{q}\right| \geq 2 \rho .
$$

Analogously, $B_{\rho}\left(p-\rho \nu_{p}\right) \cap B_{\rho}\left(q+\rho \nu_{q}\right)=\emptyset$ gives that

$$
\left|q+\rho \nu_{q}-p+\rho \nu_{p}\right| \geq 2 \rho .
$$

By adding the squares of the last two inequalities we obtain that

$$
|p-q|^{2}+2 \rho^{2}\left(\nu_{p} \cdot \nu_{q}\right) \geq 2 \rho^{2},
$$

and from (7) we get (9). From (6) and (9) we obtain (8) in $B_{r} \cap T_{p} S$. Since $|\nabla u|$ is bounded in $\bar{B}_{r} \cap T_{p} S$, then we can extend $u$ in a larger ball where $(8)$ is still satisfied. It is clear that we can choose $r=\rho$ and (7)-(9) hold.

Given $p, q \in S$ we denote by $d_{S}(p, q)$ their intrinsic distance inside $S$ and, if $A$ is an arbitrary subset of $S$, we define

$$
d_{S}(p, A)=\inf _{q \in A} d_{S}(p, q) .
$$

We have the following Lemma.

Lemma 2.2. Let $p \in S, q \in \mathcal{U}_{\rho}(p)$ and let $x$ be the orthogonal projection of $q$ onto the hyperplane $T_{p} S$. Then,

$$
|x| \leq d_{S}(p, q) \leq \rho \arcsin \frac{|x|}{\rho} .
$$

Proof. The first inequality is trivial. In order to prove the second inequality we consider the curve $\gamma:[0,1] \rightarrow S$ joining $p$ with $q$ defined by $\gamma(t)=p+t x+u(t x) \nu_{p}, t \in[0,1]$. Then

$$
\dot{\gamma}(t)=x+(\nabla u(t x) \cdot x) \nu_{p}
$$

since $x \in T_{p} S$ and by Cauchy-Schwartz inequality we obtain that

$$
|\dot{\gamma}(t)| \leq|x| \sqrt{1+|\nabla u(t x)|^{2}} .
$$


Therefore inequality (8) in Lemma 2.1 implies

$$
|\dot{\gamma}(t)| \leq \frac{\rho|x|}{\sqrt{\rho^{2}-t^{2}|x|^{2}}}
$$

Since

$$
d_{S}(p, q) \leq \int_{0}^{1}|\dot{\gamma}(t)| d t
$$

then

$$
d_{S}(p, q) \leq|x| \rho \int_{0}^{1} \frac{1}{\sqrt{\rho^{2}-t^{2}|x|^{2}}} d t
$$

which gives 10 .

Let $p \in S$ and let $u: B_{\rho} \cap T_{p} S \rightarrow S$ as in Lemma 2.1. It is well-known (see [26]) that $u$ is a classical solution to

$$
\operatorname{div}\left(\frac{\nabla u}{\sqrt{1+|\nabla u|^{2}}}\right)=n H, \quad \text { in } B_{\rho} \cap T_{p} S,
$$

where $H$ is the mean curvature of $S$ regarded as a map on $B_{\rho} \cap T_{p} S$. We notice that $\nabla u \in T_{p} S$ and the divergence is meant in local coordinates on $T_{p} S$ : if $\left\{e_{1}, \ldots, e_{n}\right\}$ is an orthonormal basis of $T_{p} S$ and $F=\sum_{i=1}^{n} F_{i} e_{i}$, then

$$
\operatorname{div} F=\sum_{i=1}^{n} \frac{\partial F_{i}}{\partial e_{i}}
$$

Moreover, (11) is uniformly elliptic once $u$ is regarded as a regular map in an open set of $\mathbb{R}^{n}$ and has bounded gradient, since

$$
|\xi|^{2} \leq \frac{\partial}{\partial \zeta_{j}}\left(\frac{\zeta_{i}}{\sqrt{1+|\zeta|^{2}}}\right) \xi_{i} \xi_{j} \leq\left(1+|\zeta|^{2}\right)|\xi|^{2} .
$$

for every $\xi=\left(\xi_{1} \ldots, \xi_{n}\right), \zeta=\left(\zeta_{1}, \ldots, \zeta_{n}\right)$ in $\mathbb{R}^{n}$.

2.1. Classical solutions to mean curvature equation. In this subsection we collect some results about classical solutions to (11) which will be used in the next sections.

Let $B_{r}$ be the ball of $\mathbb{R}^{k}$ centered at the origin and having radius $r$. Given a differentiable map $u: B_{r} \rightarrow \mathbb{R}$, we denote by $D u$ the gradient of $u$ in $\mathbb{R}^{k}$ :

$$
D u=\left(\frac{\partial u}{\partial x_{1}}, \ldots, \frac{\partial u}{\partial x_{k}}\right) .
$$

We remark that this notation differs from the one in the rest of the paper, where we use the $\nabla$ symbol to denote a vector in $\mathbb{R}^{n+1}$.

Let $H_{0}, H_{1} \in C^{0}\left(B_{r}\right)$ and $u_{0}$ and $u_{1}$ be two classical solutions of

$$
\operatorname{div}\left(\frac{D u_{j}}{\sqrt{1+\left|D u_{j}\right|^{2}}}\right)=k H_{j}, \quad \text { in } B_{r},
$$

$j=0,1$. It is well-known that (see [26])

$$
w=u_{1}-u_{0}
$$

satisfies a linear elliptic equation of the form

$$
L w=k\left(H_{1}-H_{0}\right),
$$


where

$$
L w=\sum_{i, j=1}^{k} \frac{\partial}{\partial x_{j}}\left(a^{i j}(x) \frac{\partial w}{\partial x_{i}}\right)
$$

with

$$
a^{i j}(x)=\left.\int_{0}^{1} \frac{\partial}{\partial \zeta_{j}}\left(\frac{\zeta_{i}}{\sqrt{1+|\zeta|^{2}}}\right)\right|_{\zeta=D u_{t}(x)} d t
$$

and

$$
u_{t}(x)=t u_{1}(x)+(1-t) u_{0}(x), \quad x \in B_{r} .
$$

From (12), we find that

$$
|\xi|^{2} \leq a^{i j}(x) \xi_{i} \xi_{j} \leq|\xi|^{2} \int_{0}^{1}\left(1+\left|D u_{t}(x)\right|^{2}\right) d t
$$

where we used Einstein summation convention. The following Harnack's type inequality will be one of the crucial tools for proving the stability result.

Lemma 2.3. Let $u_{j}, j=0,1$, be two classical solutions of (13), with $u_{1}-u_{0} \geq 0$ in $B_{r}$, and assume that

$$
\left\|D u_{j}\right\|_{C^{1}\left(B_{r}\right)} \leq M, \quad j=0,1,
$$

for some positive constant $M$. Then there exists a constant $K_{1}$, depending only on the dimension $k$ and $M$, such that

$$
\left\|u_{1}-u_{0}\right\|_{C^{1}\left(B_{r / 4}\right)} \leq K_{1}\left(\inf _{B_{r / 2}}\left(u_{1}-u_{0}\right)+\left\|H_{1}-H_{0}\right\|_{C^{0}\left(B_{r}\right)}\right) .
$$

Proof. We have already observed that $w=u_{1}-u_{0}$ satisfies (14) in $B_{r}$. From (16) and (17), we find that $L w$ is uniformly elliptic with continuous bounded coefficients, that is

$$
|\xi|^{2} \leq a^{i j}(x) \xi_{i} \xi_{j} \leq|\xi|^{2}\left(1+M^{2}\right),
$$

and

$$
\left|\frac{\partial}{\partial x_{j}} a^{i j}(x)\right| \leq M^{\prime}
$$

for some positive $M^{\prime}$ depending only on $M$.

From Theorems 8.17 and 8.18 in [26], we obtain the following Harnack's inequality

$$
\sup _{B_{r / 2}} w \leq C_{1}\left(\inf _{B_{r / 2}} w+\left\|H_{1}-H_{0}\right\|_{C^{0}\left(B_{r}\right)}\right) .
$$

Then we use Theorem 8.32 in [26] and obtain that

$$
|w|_{C^{1, \alpha}\left(B_{r / 4}\right)} \leq C_{2}\left(\|w\|_{C^{0}\left(B_{r / 2}\right)}+\left\|H_{1}-H_{0}\right\|_{C^{0}\left(B_{r / 2}\right)}\right),
$$

where $|\cdot|_{C^{1, \alpha}\left(B_{r / 4}\right)}$ is the $C^{1, \alpha}$ seminorm in $B_{r / 4}$, with $\alpha \in(0,1)$. By combining the last two inequalities, we obtain (18) at once.

Another crucial tool for our result is the following boundary Harnack's type inequality (or Carleson estimate, see [14]). 
Lemma 2.4. Let $E$ be a domain in $\mathbb{R}^{k}$ and let $T$ be an open set of $\partial E$ which is of class $C^{2}$. Let $u_{j} \in C^{2}(\bar{E}), j=0,1$, be two solutions of

$$
\operatorname{div}\left(\frac{D u_{j}}{\sqrt{1+\left|D u_{j}\right|^{2}}}\right)=k H_{j}, \quad \text { in } E,
$$

with $j=0,1$, satisfying $\left\|D u_{j}\right\|_{C^{1}(E)} \leq M$ for some positive $M$. Let $x_{0} \in T$ and $r>0$ be such that $B_{r}\left(x_{0}\right) \cap \partial E \subset T$, and assume that

$$
u_{1}-u_{0} \geq 0 \text { in } B_{r}\left(x_{0}\right) \cap E, \quad u_{1}-u_{0} \equiv 0 \quad \text { on } B_{r} \cap \partial E .
$$

Assume further that $e_{1}$ is the interior normal to $E$ at $x_{0}$. Then, there exists a constant $K_{2}>0$ such that

$$
\sup _{B_{r / 4}\left(x_{0}\right) \cap E}\left(u_{1}-u_{0}\right) \leq K_{2}\left(\left(u_{1}-u_{0}\right)\left(x_{0}+\frac{r}{2} e_{1}\right)+\left\|H_{1}-H_{0}\right\|_{C^{0}\left(B_{r}\right)}\right),
$$

where the constant $K_{2}$ depends only on the dimension $k, M$ and the $C^{2}$ regularity of $T$.

Proof. The proof is analogous to the one of Lemma 2.3, where we use Theorem 1.3 in [8] and Corollary 8.36 in [26] in place of Theorems $8.17,8.18$ and 8.32 in [26].

We conclude this subsection with a quantitative version of the Hopf Lemma. We start with a statement which is valid for a general second order elliptic operator of the form

$$
\mathcal{L} w=\sum_{i, j=1}^{k} a^{i j} w_{x_{i} x_{j}}+\sum_{i=1}^{k} b^{i} w_{x_{i}}
$$

satisfying the ellipticity conditions

$$
a^{i j} \zeta_{i} \zeta_{j} \geq \lambda|\zeta|^{2}, \quad \text { and } \quad\left|a^{i j}\right|,\left|b^{i}\right| \leq \Lambda, \quad i, j=1, \ldots, k,
$$

for $\lambda, \Lambda>0$.

Lemma 2.5. Let $r>0$ and $\gamma \geq 0$ be given. Assume that $w \in C^{2}\left(B_{r}\right) \cap C^{0}\left(\bar{B}_{r}\right)$ fulfills the following conditions

with $\mathcal{L}$ given by $(21)$.

$$
\mathcal{L} w \leq \gamma \quad \text { and } \quad w \geq 0 \quad \text { in } B_{r}
$$

Then, there exists a positive constant $C$ depending on $k, \lambda, \Lambda$, and upper bound on $\gamma$ such that for any $x_{0} \in \partial B_{r}$ we have that

$$
\sup _{B_{r / 2}} w \leq C\left(\frac{w\left((1-t / r) x_{0}\right)}{t}+\gamma\right), \quad \text { for any } 0<t \leq r / 2 .
$$

Moreover, if $w\left(x_{0}\right)=0$ then we have that

$$
\sup _{B_{r / 2}} w \leq C\left(\frac{\partial w\left(x_{0}\right)}{\partial \nu}+\gamma\right)
$$

where $\nu$ denotes the inward normal to $\partial B_{r}$.

Proof. In the annulus $A=B_{r} \backslash \bar{B}_{r / 2}$, we consider the auxiliary function

$$
v(x)=\left(\min _{\bar{B}_{r / 2}} w\right) \frac{e^{-\alpha|x|^{2}}-e^{-\alpha r^{2}}}{e^{-\alpha(r / 2)^{2}}-e^{-\alpha r^{2}}}+e^{\beta|x|^{2}}-e^{\beta r^{2}},
$$

where

$$
\alpha=\frac{(k+r \sqrt{k}) \Lambda}{2 \lambda^{2}}, \quad \beta=\gamma\left[k \lambda-\sqrt{k} \Lambda r+\sqrt{(k \lambda-\sqrt{k} \Lambda r)^{2}+\gamma \lambda r^{2}}\right]^{-1} .
$$


Here, the constants $\alpha$ and $\beta$ are chosen in such a way that $v$ satisfies

$$
\mathcal{L} v \geq \gamma .
$$

We notice that

$$
\frac{v\left((1-t / r) x_{0}\right)}{t} \geq \frac{\alpha r e^{-\alpha r^{2}}}{e^{-\alpha(r / 2)^{2}}-e^{-\alpha r^{2}}}\left(\min _{\bar{B}_{r / 2}} w\right)-2 \beta r e^{\beta r^{2}} .
$$

Since $v=0$ on $\partial B_{r}$ and $v \leq \min _{\partial B_{r / 2}} w$ on $\partial B_{r / 2}$, we have that the function $w-v$ satisfies the following conditions

$$
\begin{cases}\mathcal{L}(w-v) \leq 0, & \text { in } A, \\ w-v \geq 0, & \text { on } \partial A .\end{cases}
$$

Hence, by maximum principle we have that $w-v \geq 0$ in $\bar{A}$, and from (25) we obtain that

$$
\min _{\bar{B}_{r / 2}} w \leq \frac{e^{3 \alpha r^{2} / 4}-1}{\alpha r}\left(\frac{w\left((1-t / r) x_{0}\right)}{t}+2 \beta r e^{\beta r^{2}}\right),
$$

for $0<t<r / 2$. As in the proof of Lemma 2.3, we use Theorems 8.17 and 8.18 in [26] to get

$$
\max _{\bar{B}_{r / 2}} w \leq C_{1}\left(\min _{\bar{B}_{r / 2}} w+\gamma\right),
$$

and from (26) we obtain (23) and (24).

We will use Lemma 2.5 in the following form.

Lemma 2.6. Let $E, T, u_{0}, u_{1}, M$, and $x_{0}$ be as in Lemma 2.4, with

$$
u_{1}-u_{0} \geq 0 \quad \text { in } E \text {. }
$$

Assume that there exists $B_{r}(c) \subset E$ with $x_{0} \in \partial B_{r}(c) \cap T$. Let

$$
\ell=\frac{c-x_{0}}{r} \text {. }
$$

Then, there exists a constant $K_{3}$ such that

$$
\left\|u_{1}-u_{0}\right\|_{C^{1}\left(B_{r / 4}(c)\right)} \leq K_{3}\left(\frac{\left(u_{1}-u_{0}\right)\left(x_{0}+t \ell\right)}{t}+\left\|H_{1}-H_{0}\right\|_{C^{0}\left(B_{r}(c)\right)}\right),
$$

for every $t \in(0, r / 2)$, and

$$
\left\|u_{1}-u_{0}\right\|_{C^{1}\left(B_{r / 4}(c)\right)} \leq K_{3}\left(\frac{\partial\left(u_{1}-u_{0}\right)}{\partial \ell}\left(x_{0}\right)+\left\|H_{1}-H_{0}\right\|_{C^{0}\left(B_{r}(c)\right)}\right),
$$

for $t=0$. The constant $K_{3}$ depends only on the dimension $k$, on $M$, and $\rho$, and upper bound on $\left\|H_{1}-H_{0}\right\|_{C^{0}\left(B_{r}(c)\right)}$.

Proof. As we have shown in the proof of Lemma 2.3, $w=u_{1}-u_{0}$ satisfies (14), which is uniformly elliptic. Moreover, we notice that, by letting

$$
\gamma=\left\|H_{1}-H_{0}\right\|_{C^{0}\left(B_{r}(c)\right)}
$$

we have that

$$
L w \leq \gamma
$$

Hence, we can apply Lemma 2.5 and, by using Lemma 2.3, we conclude. 
2.2. The symmetry result of Alexandrov. In order to make the paper self-contained, we give a sketch of the proof of the Soap Bubble Theorem by Alexandrov. This will be the occasion to set up some necessary notation.

Let $S$ be a $C^{2}$ regular, connected, closed hypersurface embedded in $\mathbb{R}^{n+1}, n \geq 1$, and let $\Omega$ be the relatively compact domain of $\mathbb{R}^{n+1}$ bounded by $S$. Let $\omega \in \mathbb{R}^{n+1}$ be a unit vector and $\lambda \in \mathbb{R}$ be a parameter. For an arbitrary set $A$, we define the following objects:

$$
\begin{array}{ll}
\pi_{\lambda}=\left\{\xi \in \mathbb{R}^{n+1}: \xi \cdot \omega=\lambda\right\} & \text { a hyperplane orthogonal to } \omega, \\
A^{\lambda}=\{p \in A: p \cdot \omega>\lambda\} & \text { the right-hand cap of } A, \\
\xi^{\lambda}=\xi-2(\xi \cdot \omega-\lambda) \omega & \text { the reflection of } \xi \text { about } \pi_{\lambda}, \\
A_{\lambda}=\left\{p \in \mathbb{R}^{n+1}: p^{\lambda} \in A^{\lambda}\right\} & \text { the reflected cap about } \pi_{\lambda}, \\
\hat{A}_{\lambda}=\{p \in A: p \cdot \omega<\lambda\} & \text { the portion of } A \text { in the left-hand half plane. }
\end{array}
$$

Set $\mathcal{M}=\max \{p \cdot \omega: p \in S\}$, the extent of $S$ in the direction $\omega$; if $\lambda<\mathcal{M}$ is close to $\mathcal{M}$, the reflected cap $\Omega_{\lambda}$ is contained in $\Omega$. Set

$$
m=\inf \left\{\mu: \Omega_{\lambda} \subset \Omega \text { for all } \lambda \in(\mu, \mathcal{M})\right\} .
$$

Then for $\lambda=m$ at least one of the following two cases occurs:

(i) $S_{m}$ becomes internally tangent to $S$ at some point $p \in S \backslash \pi_{m}$;

(ii) $\pi_{m}$ is orthogonal to $S$ at some point $p \in S \cap \pi_{m}$.

Theorem A (Alexandrov Soup Bubble Theorem). Let $S$ be a $C^{2}$-regular, closed, connected hypersurface embedded in $\mathbb{R}^{n+1}$. If the mean curvature $H$ of $S$ is constant, then $S$ is a sphere.

Proof. Let $\omega$ be a fixed direction. We apply the method of moving planes in the direction $\omega$ and we find a critical position for $\lambda=m$.

If Case (i) occurs, then we locally write $S_{m}$ and $S$ as graphs of function $u_{1}$ and $u_{0}$, respectively, over $B_{r} \cap T_{p} S$ (which coincides with $T_{p} S_{m}$ ), where $p$ is the tangency point. It is clear that $w=u_{1}-u_{0}$ is non-negative and, since $H$ is constant, we have that $w$ satisfies

$$
L w=0, \quad \text { in } B_{r} \cap T_{p} S,
$$

for some $r>0$, and where $L$ is given by $(15)$. Since $w(0)=0$, by the strong maximum principle we obtain that $w \equiv 0$ in $B_{r} \cap T_{p} S$, that is $S$ and $S_{m}$ coincides in an open neighborhood of $p$.

If Case (ii) occurs, then we locally write $S_{m}$ and $S$ as graphs of function $u_{1}$ and $u_{0}$, respectively, over $T_{p} S \cap\{x \cdot \omega \leq m\}$. As for case (i), we find that there exists $r>0$ such that

$$
\begin{cases}L w=0, & \text { in } \quad B_{r} \cap T_{p} S \cap\{x \cdot \omega<m\}, \\ w=0, & \text { on } \quad B_{r} \cap T_{p} S \cap\{x \cdot \omega=m\} .\end{cases}
$$

Since $\nabla w(0)=0$ and from the Hopf Lemma (see for instance [26]) we obtain that $w \equiv 0$ in $B_{r} \cap T_{p} S \cap\{x \cdot \omega \leq m\}$.

Hence, in both cases (i) and (ii) we have that the set of tangency points (that is those points for which case (i) or (ii) occur) is open. Since it is also closed and non-empty we must have that $S_{m}=\hat{S}_{m}$, that is $S$ is symmetric about the hyperplane $\pi_{m}$. Since $\omega$ is arbitrary, we find that $S$ is symmetric in every direction.

Up to a translation, we can assume that the origin $O$ is the center of mass of $S$. Since $O$ belongs to every axis of symmetry and every rotation can be written as a composition of reflections, we have that $S$ is invariant under rotations, which implies that $S$ is a sphere. 
2.3. Curvatures of projected surfaces. Before giving the results of this subsection, we need to recall some basic facts about hypersurfaces in $\mathbb{R}^{n+1}$, in particular about the interplay between the normal and the principal curvatures. Let $U$ be an orientable hypersurface of class $C^{2}$ embedded in $\mathbb{R}^{n+1}$ (which in the proof of Theorem 1.1 will be an open set of the surface $S$ ). The choice of an orientation on $U$ is equivalent to the choice of a Gauss map $\nu: U \rightarrow \mathbb{S}^{n}$ (in this general context there is no a canonical orientation). Fixed a point $q \in U$, we denote by $W_{q}: T_{q} U \rightarrow T_{q} U$ the shape operator $W_{q}=-d \nu_{q} . W_{q}$ is a symmetric operator and its eigenvalues $\kappa_{i}(q)$ are the principal curvatures of $U$ at $q$. We assume that $\kappa_{1}(q) \leq \kappa_{2}(q) \leq \cdots \leq \kappa_{n}(q)$. The first and the last principal curvature can be obtained as minimum and maximum of the normal curvature. Here we recall that, given a non-zero vector $v \in T_{q} U$, its normal curvature $\kappa(q, v)$ is defined as

$$
\kappa(q, v)=\frac{1}{|v|^{2}} W_{q}(v) \cdot v .
$$

$\kappa(q, v)$ can be alternatively written in term of curves as

$$
\kappa(q, v)=\frac{1}{|\dot{\alpha}(0)|^{2}} \nu_{\alpha(0)} \cdot \ddot{\alpha}(0)
$$

where $\alpha: I \rightarrow U$ is an arbitrary curve satisfying $\alpha(0)=0, \dot{\alpha}(0)=v$.

In order to perform a quantitive study of the moving planes, we need to handle the following situation: given a hypersurface $U$ of class $C^{2}$ in $\mathbb{R}^{n+1}$, we consider its intersection $U^{\prime}$ with an affine hyperplane $\pi_{1}$ (in the proof of Theorem $4.1 \pi_{1}$ will be the critical hyperplane in the direction $\omega$ ). If $\pi_{1}$ intersects $U$ transversally, $U^{\prime}=U \cap \pi_{1}$ is a hypersurface of class $C^{2}$ of $\pi_{1}$ and we consider its projection $U^{\prime \prime}$ onto another hyperplane $\pi_{2}$ of $\mathbb{R}^{n+1}$ (which will be the tangent hyperplane to the reflected cap at some point which is close to the critical hyperplane). An example in $\mathbb{R}^{3}$ is shown in Figure 1. The next two propositions allow us to control the principal curvature of $U^{\prime \prime}$ in terms of the principal curvature of $U$ and the normal vectors to $\omega_{1}$ and $\omega_{2}$.

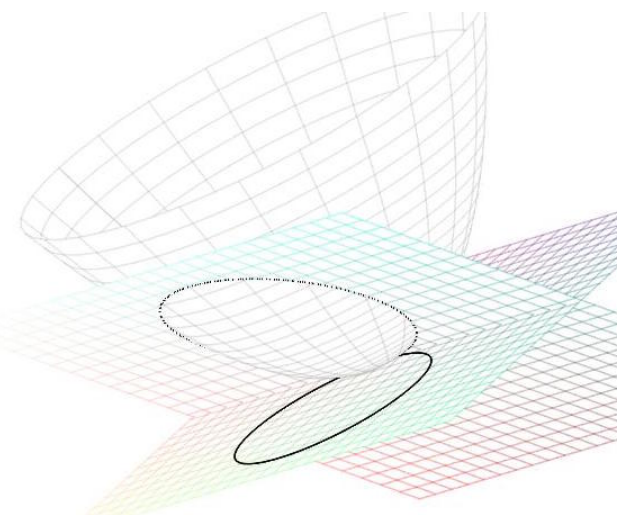

FiguRE 1. In the figure $U$ is the parabololid $z=x^{2}+y^{2}, \pi_{1}$ is the affine plane $z=2+8 y$ and $\pi_{2}$ is the plane $z=0$. In this case $U^{\prime}$ is the dashed ellipse in $\pi_{1}$, while $U^{\prime \prime}$ is the circle projected in $\pi_{2}$.

Proposition 2.7. Let $U$ be an orientable hypersurface of class $C^{2}$ embedded in $\mathbb{R}^{n+1}$ with principal curvatures $\kappa_{j}, j=1, \ldots, n$, and Gauss map $\nu$. Let $\pi$ be an hyperplane of $\mathbb{R}^{n+1}$ intersecting $U$ transversally and let $U^{\prime}=U \cap \pi$. Then $U^{\prime}$ is an orientable hypersurface of class 
$C^{2}$ embedded in $\pi$ and, once a Gauss map $\nu^{\prime}: U^{\prime} \rightarrow \mathbb{S}^{n-1}$ is fixed, its principal curvatures $\kappa_{i}^{\prime}$ satisfy

$$
\frac{1}{\nu_{q} \cdot \nu_{q}^{\prime}} \kappa_{1}(q) \leq \kappa_{i}^{\prime}(q) \leq \frac{1}{\nu_{q} \cdot \nu_{q}^{\prime}} \kappa_{n}(q)
$$

for every $q \in U^{\prime}$ and $i=1, \ldots, n-1$.

Proof. First of all we observe that $U^{\prime}$ is of class $C^{2}$ by the implicit function theorem and it is orientable since the map $\nu^{\prime}: U^{\prime} \rightarrow \mathbb{S}^{n-1}$ defined by

$$
\nu_{q}^{\prime}=-*\left(*\left(\nu_{q} \wedge \omega\right) \wedge \omega\right),
$$

is a Gauss map on $U^{\prime}$, where by $*$ we denote the Hodge "star" operator in $\mathbb{R}^{n+1}$ computed with respect to the standard metric and the standard orientation.

In order to prove (31): fix $q \in U^{\prime}$ and consider an arbitrary unitary vector $v \in T_{q} U^{\prime}$. Let $\kappa(q, v)$ be normal curvature of $(q, v)$ in $U$. Then

$$
\kappa(q, v)=\nu_{q} \cdot \ddot{\alpha}(0)
$$

where $\alpha$ is an arbitrary smooth curve in $U^{\prime}$ parametrized by arc length and such that $\alpha(0)=q$ and $\dot{\alpha}(0)=v$. Since $\nu_{q}$ is orthogonal to $T_{q} U^{\prime}$, it belongs to the plane generated by $\omega$ and $\nu_{q}^{\prime}$ and we can write

Therefore

$$
\nu_{q}=\left(\nu_{q}^{\prime} \cdot \omega\right) \omega+\left(\nu_{q} \cdot \nu_{q}^{\prime}\right) \nu_{q}^{\prime}
$$

$$
\kappa(q, v)=\left(\nu_{q} \cdot \ddot{\alpha}(0)\right)=\left(\nu_{q} \cdot \nu_{q}^{\prime}\right)\left(\nu_{q}^{\prime} \cdot \ddot{\alpha}(0)\right)=\left(\nu_{q} \cdot \nu_{q}^{\prime}\right) \kappa^{\prime}(q, v),
$$

where $\kappa^{\prime}(q, v)$ is the normal curvature of $U^{\prime}$ in $(q, v)$, and the claim follows.

Note that in Proposition 2.7, if we chose as Gauss map the one defined as in (32) we have

$$
\left(\nu_{q} \cdot \nu_{q}^{\prime}\right)=-*\left(*\left(\nu_{q} \wedge \omega\right) \wedge \omega\right) \cdot \nu_{q}=-*\left(\nu_{q} \wedge \omega\right) \wedge \omega \cdot * \nu_{q} .
$$

Now we choose the positive oriented orthonormal basis of $\mathbb{R}^{n+1}\left\{e_{1}, \ldots, e_{n}, \nu_{q}\right\}$ where the first $n$-vectors are an othonormal basis of $T_{q} U$. Then we have

$$
*\left(\nu_{q} \wedge \omega\right)=-\left(\omega \cdot e_{n}\right) e_{1} \wedge \cdots \wedge e_{n-1}, \quad * \nu_{q}=e_{1} \wedge \cdots \wedge e_{n},
$$

and

$$
\nu_{q} \cdot \nu_{q}^{\prime}=\left(\omega \cdot e_{n}\right)\left(e_{1} \wedge \cdots \wedge e_{n-1} \wedge \omega\right) \cdot\left(e_{1} \wedge \cdots \wedge e_{n}\right)=\left(\omega \cdot e_{n}\right)^{2}=1-\left(\nu_{q} \cdot \omega\right)^{2},
$$

i.e.

$$
\nu_{q} \cdot \nu_{q}^{\prime}=1-\left(\nu_{q} \cdot \omega\right)^{2} .
$$

Therefore, when $\nu_{q}^{\prime}$ is given by (32), 31) reads as

$$
\frac{1}{1-\left(\nu_{q} \cdot \omega\right)^{2}} \kappa_{1}(q) \leq \kappa_{i}^{\prime}(q) \leq \frac{1}{1-\left(\nu_{q} \cdot \omega\right)^{2}} \kappa_{n}(q) .
$$

for $i=1, \ldots, n-1$.

Proposition 2.8. Let $\omega_{1}$ and $\omega_{2}$ be unit vectors in $\mathbb{R}^{n+1}$, denote by $\pi_{1}$ an hyperplane orthogonal to $\omega_{1}$, and let $\pi_{2}$ be the hyperplane orthogonal to $\omega_{2}$ passing through the origin of $\mathbb{R}^{n+1}$. Let $U^{\prime}$ be a $C^{2}$ regular oriented hypersurface of $\pi_{1}$ such that $\omega_{2}$ is not tangent to $U^{\prime}$ at any point. Denote by $\kappa_{i}^{\prime}$, for $i=1, \ldots, n-1$, the principal curvatures of $U^{\prime}$ and denote by $\nu^{\prime}$ the normal 
vector to $U^{\prime}$. Then the orthogonal projection $U^{\prime \prime}$ of $U^{\prime}$ onto $\pi_{2}$ is a $C^{2}$-regular hypersurface of $\pi_{2}$ with a canonical orientation. Moreover, for any $q \in U^{\prime}$ we have

$$
\left|\kappa_{i}^{\prime \prime}(\operatorname{pr}(q))\right| \leq \frac{\left|\omega_{1} \cdot \omega_{2}\right|}{\left[\left(\omega_{1} \cdot \omega_{2}\right)^{2}+\left(\omega_{2} \cdot \nu_{q}^{\prime}\right)^{2}\right]^{\frac{3}{2}}} \max \left\{\left|\kappa_{1}^{\prime}(q)\right|,\left|\kappa_{n-1}^{\prime}(q)\right|\right\},
$$

for every $i=1, \ldots, n-1$, where $\operatorname{pr}(q)$ is the projection of $q$ onto $\pi_{2}$ and $\left\{\kappa_{i}^{\prime \prime}\right\}$ are the principal curvature of $U^{\prime \prime}$.

Proof. If $X$ is a local positive oriented parametrisation of $U^{\prime}$, then $Y=X-\left(X \cdot \omega_{2}\right) \omega_{2}$ is a local parametrisation of $U^{\prime \prime}$, and

$$
\nu^{\prime \prime} \circ Y:=\operatorname{vers}\left(*\left(Y_{1} \wedge Y_{2} \wedge \cdots \wedge Y_{n-1} \wedge \omega_{2}\right)\right)
$$

defines a Gauss map for $U^{\prime \prime}$, where $Y_{k}$ is the $k^{t h}$ derivative of $Y$ with respect to the coordinates of its domain. Therefore $U^{\prime \prime}$ is a $C^{2}$-regular hypersurface of $\pi_{2}$ oriented by the map $\nu^{\prime \prime}$.

Now we prove inequalities (35). Fix a point $q \in U^{\prime}$ and let $\operatorname{pr}(q)=q-\left(q \cdot \omega_{2}\right) \omega_{2}$ be its projection onto $U^{\prime \prime}$. Let $X$ be a local positive oriented parametrization of $U^{\prime}$ around $q$ and $Y=X-\left(X \cdot \omega_{2}\right) \omega_{2}$ be the induced parametrization of $U^{\prime \prime}$ around $\operatorname{pr}(q)$. Let $\beta:(-\delta, \delta) \rightarrow U^{\prime \prime}$ be an arbitrary regular curve contained in $U^{\prime \prime}$ such that $\beta(0)=\operatorname{pr}(q)$ and let

$$
v=\frac{\dot{\beta}(0)}{|\dot{\beta}(0)|}, \quad g=\frac{1}{|\dot{\beta}|^{2}} \nu_{\beta}^{\prime \prime} \cdot \ddot{\beta} .
$$

Then

$$
g(0)=\kappa^{\prime \prime}(\operatorname{pr}(q), v),
$$

where $\kappa^{\prime \prime}(\operatorname{pr}(q), v)$ is the normal curvature of $U^{\prime \prime}$ at $(q, v)$. The curve $\beta$ can be seen as the projection of a regular curve $\alpha$ in $U^{\prime}$ passing through $p$. Since $\nu_{\beta}^{\prime \prime}$ is orthogonal to $\omega_{2}$ we have

$$
g=\frac{1}{|\dot{\beta}|^{2}} \nu_{\beta}^{\prime \prime} \cdot \ddot{\alpha}
$$

Note that, since

$$
Y_{k}=X_{k}-\left(X_{k} \cdot \omega_{2}\right) \omega_{2}
$$

then we have

$$
\nu^{\prime \prime} \circ Y=\operatorname{vers}\left(*\left(X_{1} \wedge X_{2} \wedge \cdots \wedge X_{n-1} \wedge \omega_{2}\right)\right)
$$

and

$$
g=\frac{\left(*\left(X_{1}(\tilde{\alpha}) \wedge \cdots \wedge X_{n-1}(\tilde{\alpha}) \wedge \omega_{2}\right) \cdot \ddot{\alpha}\right.}{\left.|\dot{\beta}|^{2} \mid X_{1}(\tilde{\alpha}) \wedge \cdots \wedge X_{n-1}(\tilde{\alpha}) \wedge \omega_{2}\right) \mid} .
$$

Now, it is simply to prove that

$$
\left(*\left(X_{1}(\tilde{\alpha}) \wedge \cdots \wedge X_{n-1}(\tilde{\alpha}) \wedge \omega_{2}\right) \cdot \ddot{\alpha}=\left(\omega_{1} \cdot \omega_{2}\right) *\left(X_{1}(\tilde{\alpha}) \wedge \cdots \wedge X_{n-1}(\tilde{\alpha}) \wedge \omega_{1}\right) \cdot \ddot{\alpha},\right.
$$

and therefore

$$
g=\frac{\omega_{1} \cdot \omega_{2}}{|\dot{\beta}|^{2}} \frac{\left(*\left(X_{1}(\tilde{\alpha}) \wedge \cdots \wedge X_{n-1}(\tilde{\alpha}) \wedge \omega_{1}\right)\right) \cdot \ddot{\alpha}}{\left.\mid X_{1}(\tilde{\alpha}) \wedge \cdots \wedge X_{n-1}(\tilde{\alpha}) \wedge \omega_{2}\right) \mid}
$$

which implies

$$
g=\left(\nu_{\alpha}^{\prime} \cdot \ddot{\alpha}\right) \frac{\omega_{1} \cdot \omega_{2}}{|\dot{\beta}|^{2}} \frac{\left|X_{1}(\tilde{\alpha}) \wedge \cdots \wedge X_{n-1}(\tilde{\alpha}) \wedge \omega_{1}\right|}{\left|X_{1}(\tilde{\alpha}) \wedge \cdots \wedge X_{n-1}(\tilde{\alpha}) \wedge \omega_{2}\right|} .
$$

We may assume that $\alpha$ is parametrised by arc length and so

$$
|\dot{\beta}|^{2}=1-\left(\dot{\alpha} \cdot \omega_{2}\right)^{2}
$$


which implies

$$
g=\left(\nu_{\alpha}^{\prime} \cdot \ddot{\alpha}\right) \frac{\omega_{1} \cdot \omega_{2}}{1-\left(\dot{\alpha} \cdot \omega_{2}\right)^{2}} \frac{\left|X_{1}(\tilde{\alpha}) \wedge \cdots \wedge X_{n-1}(\tilde{\alpha}) \wedge \omega_{1}\right|}{\left|X_{1}(\tilde{\alpha}) \wedge \cdots \wedge X_{n-1}(\tilde{\alpha}) \wedge \omega_{2}\right|} .
$$

Moreover a standard computation yields that

$$
\frac{\left|X_{1}(\tilde{\alpha}) \wedge \cdots \wedge X_{n-1}(\tilde{\alpha}) \wedge \omega_{1}\right|}{\left|X_{1}(\tilde{\alpha}) \wedge \cdots \wedge X_{n-1}(\tilde{\alpha}) \wedge \omega_{2}\right|}=\frac{1}{\left(1-\left|\omega_{2}-\left(\omega_{2} \cdot \nu_{\alpha}^{\prime}\right) \nu_{\alpha}^{\prime}\right|^{2}\right)^{1 / 2}},
$$

and hence

$$
g(0)=\kappa^{\prime}(q, \dot{\alpha}(0)) \frac{\omega_{1} \cdot \omega_{2}}{\left(1-\left|\omega_{2}-\left(\omega_{2} \cdot \nu_{q}^{\prime}\right) \nu_{q}^{\prime}\right|^{2}\right)^{1 / 2}} \frac{1}{1-\left(\dot{\alpha}(0) \cdot \omega_{2}\right)^{2}},
$$

where $\kappa^{\prime}(q, \dot{\alpha}(0))$ is the normal curvature of $U^{\prime}$ at $(q, \dot{\alpha}(0))$. Therefore

$$
\kappa^{\prime \prime}(\operatorname{pr}(q), v)=\kappa^{\prime}(q, \dot{\alpha}(0)) \frac{\omega_{1} \cdot \omega_{2}}{\left(1-\left|\omega_{2}-\left(\omega_{2} \cdot \nu_{q}^{\prime}\right) \nu_{q}^{\prime}\right|^{2}\right)^{1 / 2}} \frac{1}{1-\left(\dot{\alpha}(0) \cdot \omega_{2}\right)^{2}} .
$$

In particular

$$
\kappa_{1}^{\prime \prime}(\operatorname{pr}(q))=\kappa_{1}^{\prime}(q) \frac{\omega_{1} \cdot \omega_{2}}{\left(1-\left|\omega_{2}-\left(\omega_{2} \cdot \nu_{q}^{\prime}\right) \nu_{q}^{\prime}\right|^{2}\right)^{1 / 2}} \inf _{v \in \mathbb{S}_{q}^{n-1}} \frac{1}{1-\left(v \cdot \omega_{2}\right)^{2}},
$$

and

$$
\kappa_{n-1}^{\prime \prime}(\operatorname{pr}(q))=\kappa_{n-1}^{\prime}(p) \frac{\omega_{1} \cdot \omega_{2}}{\left(1-\left|\omega_{2}-\left(\omega_{2} \cdot \nu_{q}^{\prime}\right) \nu_{q}^{\prime}\right|^{2}\right)^{1 / 2}} \sup _{v \in \mathbb{S}_{q}^{n-1}} \frac{1}{1-\left(v \cdot \omega_{2}\right)^{2}},
$$

where $\mathbb{S}_{q}^{n-1}=\left\{v \in T_{q} U^{\prime}:|v|=1\right\}$. Now if $v \in \mathbb{S}_{q}^{n-1}$, we have

$$
1-\left(v \cdot \omega_{2}\right)^{2} \leq 1-\left|\omega_{2}-\left(\omega_{2} \cdot \nu_{q}^{\prime}\right) \nu_{q}^{\prime}\right|^{2} .
$$

Therefore

$$
\left|\kappa_{i}^{\prime \prime}(\operatorname{pr}(q))\right| \leq \frac{\left|\omega_{1} \cdot \omega_{2}\right|}{\left(1-\left|\omega_{2}-\left(\omega_{2} \cdot \nu_{q}^{\prime}\right) \nu_{q}^{\prime}\right|^{2}\right)^{3 / 2}} \max \left\{\left|\kappa_{1}^{\prime}(q)\right|,\left|\kappa_{n-1}^{\prime}(q)\right|\right\},
$$

for every $i=1, \ldots, n-1$. Finally, since $\mathbb{R}^{n+1}=T_{q} U^{\prime} \oplus\left\langle\omega_{1}\right\rangle \oplus\left\langle\nu_{q}^{\prime}\right\rangle$, we have

$$
1-\left|\omega_{2}-\left(\omega_{2} \cdot \nu_{q}^{\prime}\right) \nu_{q}^{\prime}\right|^{2}=\left(\omega_{1} \cdot \omega_{2}\right)^{2}+\left(\omega_{2} \cdot \nu_{q}^{\prime}\right)^{2}
$$

and the claim follows.

\section{Technical Lemmas}

Let $S$ be a connected closed $C^{2}$ regular hypersurface embedded in $\mathbb{R}^{n+1}$ and let $\rho$ be the radius of the uniform touching sphere.

Let $S_{m}$ and $\pi_{m}$ be as in 29 and let $\partial S_{m}=S \cap \pi_{m}$. It will be useful to define the following set

$$
S_{m}^{\delta}=\left\{p \in S_{m}: d_{S}\left(p, \partial S_{m}\right)>\delta\right\}
$$

for $\delta>0$.

Lemma 3.1. Let $0<\delta<\rho$ and set $\sigma=\rho \sin (\delta / \rho)$. Then the following facts hold:

(i) For any $p \in S_{m}^{\delta}$ we have $\mathcal{U}_{\sigma}(p) \subset S_{m}$.

(ii) For any $q \in S_{m} \backslash S_{m}^{\delta}$ there exists $p \in \partial S_{m}$ and $x \in B_{\delta} \cap T_{p} S$ such that

$$
q=p+x+u(x) \nu_{p}
$$

Here $u$ and $\mathcal{U}$ are as in (5).

Proof. 
(i) Let $x \in B_{\sigma} \cap T_{p} S$ and let $q=p+x+u(x) \nu_{p}$. Since

$$
d_{S}\left(q, \partial S_{m}\right) \geq d_{S}\left(p, \partial S_{m}\right)-d_{S}(p, q),
$$

(10) implies

$$
d_{S}\left(q, \partial S_{m}\right) \geq \delta-\rho \arcsin \frac{|x|}{\rho} .
$$

The assumption $|x|<\sigma$ implies the thesis.

(ii) Let $p \in \partial S_{m}$ be such that $d_{S}\left(q, \partial S_{m}\right)=d_{S}(p, q)$, and let $x$ be the orthogonal projection of $q$ onto $T_{p} S$. Since $|x| \leq d_{S}(p, q)<\delta$ and $\delta<\rho$, then $|x|<\rho$ and Lemma 2.1 implies the statement.

In the next lemma we show that any two points in $S_{m}^{\delta}$ can be joined by a piecewise geodesic curve and we give a bound on its length. An analogous lemma was proved in [1] in the special case when $S_{m}^{\delta}$ is contained in a hyperplane.

Lemma 3.2. Let $0<\delta<\rho$, and set

$$
L=\frac{|S| 2^{n}}{\omega_{n} \delta^{n}}
$$

where $\omega_{n}$ is volume of the unit ball in $\mathbb{R}^{n}$. Let $p, q$ be in a connected component of $S_{m}^{\delta}$. Then there exists a piecewise geodesic path $\gamma:[0,1] \rightarrow S_{m}^{\delta / 2}$ satisfying $\gamma(0)=p$ and $\gamma(1)=q$ and with length bounded by L. Moreover, $\gamma$ can be built by joining $\mathcal{N}$ minimal geodesics of length $\delta$, with

$$
\mathcal{N} \leq L
$$

and one minimal geodesic of length less or equal than $\delta$.

Proof. Let $p, q$ be in a connected component of $S_{m}^{\delta}$. We can join $p$ and $q$ by a path $\tilde{\gamma}:[0,1] \rightarrow S_{m}^{\delta}$ such that $\tilde{\gamma}(0)=p$ and $\tilde{\gamma}(1)=q$. Given a point $z_{0} \in S$, we denote by $D_{r}\left(z_{0}\right)$ the set of points on $S$ with intrinsic distance from $z_{0}$ less than $r$, i.e.

$$
D_{r}\left(z_{0}\right)=\left\{z \in S: d_{S}\left(z, z_{0}\right)<r\right\} .
$$

When $r<\rho, 10$ implies

$$
\left|D_{r}(p)\right| \geq \omega_{n} r^{n} .
$$

Then we consider the increasing sequence $\left\{t_{0}, t_{1}, \ldots, t_{I}\right\}$ in $[0,1]$ recursively defined as follows: $t_{0}=0$, and

$$
t_{i+1}=\inf \left\{t \in[0,1]: D_{\delta / 2}(\tilde{\gamma}(s)) \cap \bigcup_{j=0}^{i} D_{\delta / 2}\left(\tilde{\gamma}\left(t_{j}\right)\right)=\emptyset, \forall s \in[t, 1]\right\}
$$

if

$$
\left\{t \in[0,1]: D_{\delta / 2}(\tilde{\gamma}(s)) \cap \bigcup_{j=0}^{i} D_{\delta / 2}\left(\tilde{\gamma}\left(t_{j}\right)\right)=\emptyset \forall s \in[t, 1]\right\} \text { is non-empty, }
$$

and $t_{i+1}=t_{I}$, otherwise. Therefore $\left\{t_{0}, t_{1}, \ldots, t_{I}\right\}$ is an increasing sequence in $[0,1]$ satisfying

$$
D_{\delta / 2}\left(\tilde{\gamma}\left(t_{i}\right)\right) \cap D_{\delta / 2}\left(\tilde{\gamma}\left(t_{j}\right)\right)=\emptyset, \quad \text { for } i \neq j, i, j=0, \ldots, I
$$

and

$$
D_{\delta / 2}\left(\tilde{\gamma}\left(t_{i}\right)\right) \subset S_{m}^{\delta / 2}, \quad i=0, \ldots, I
$$


We complete the sequence by adding $t_{I+1}=1$ as last term. Since

$$
\left|\bigcup_{i=0}^{I} D_{\delta / 2}\left(\tilde{\gamma}\left(t_{i}\right)\right)\right| \leq|S|
$$

from (41) and (43) we obtain that

$$
I+1 \leq \frac{2^{n}}{\omega_{n} \delta^{n}}|S|
$$

From (42), it is clear that

$$
\bar{D}_{\delta / 2}\left(\tilde{\gamma}\left(t_{i}\right)\right) \cap\left(\bigcup_{j=0}^{i-1} \bar{D}_{\delta / 2}\left(\tilde{\gamma}\left(t_{j}\right)\right)\right) \neq \emptyset
$$

for every $i=1, \ldots, I$. Let

$$
\sigma(i)=\max \left\{j>i: \bar{D}_{\delta / 2}\left(\tilde{\gamma}\left(t_{i}\right)\right) \cap \bar{D}_{\delta / 2}\left(\tilde{\gamma}\left(t_{j}\right)\right) \neq \emptyset\right\} .
$$

Then we set $\sigma^{2}(i)=\sigma(\sigma(i)), \sigma^{3}(i)=\sigma(\sigma(\sigma(i)))$ and so on, and fix $\tau \in \mathbb{N}$ such that $\sigma^{\tau}(0)=I$. We define $\gamma_{1}$ as a minimal geodesic joining $p$ and $\tilde{\gamma}\left(t_{\sigma(0)}\right)$ such that

$$
\gamma_{1} \subset \bar{D}_{\delta / 2}(p) \cup \bar{D}_{\delta / 2}\left(\tilde{\gamma}\left(t_{\sigma(0)}\right)\right) ;
$$

for $i=2, \ldots, \tau$, we let $\gamma_{i}$ be a minimal geodesic joining $\tilde{\gamma}\left(t_{\sigma^{i}(0)}\right)$ and $\tilde{\gamma}\left(t_{\sigma^{i+1}(0)}\right)$ and such that

$$
\gamma_{i} \subset \bar{D}_{\delta / 2}\left(\tilde{\gamma}\left(t_{\sigma^{i}(0)}\right)\right) \cup \bar{D}_{\delta / 2}\left(\tilde{\gamma}\left(t_{\sigma^{i+1}(0)}\right)\right) .
$$

Moreover, we let $\gamma_{\tau+1}$ be a minimal geodesic joining $\tilde{\gamma}\left(t_{I}\right)$ and $q$ and such that

$$
\gamma_{i} \subset \bar{D}_{\delta / 2}\left(\tilde{\gamma}\left(t_{\sigma^{i}(0)}\right)\right) \cup \bar{D}_{\delta / 2}(q)
$$

Let $\gamma$ be the piecewise geodesic obtained by the union of $\gamma_{1}, \ldots, \gamma_{\tau+1}$. It is clear that each $\gamma_{i}$ has length equal to $\delta$ for $i=1, \ldots, \tau$, and less or equal than $\delta$ for $i=\tau+1$. Since $\tau \leq I$, from (44) we obtain that

$$
\text { length }(\gamma) \leq(\tau+1) \delta \leq \frac{2^{n}}{\omega_{n} \delta^{n}}|S|,
$$

which implies (39) and (40), and the proof is complete.

It will be useful to define the following two numbers:

$$
\varepsilon_{0}=\min \left(\frac{1}{2}, \frac{\rho}{16 L} \sin \frac{\delta}{2 \rho}\right)
$$

and

$$
N_{0}=1+\left[\log _{\left(1-\varepsilon_{0}\right)} \frac{1}{2}\right],
$$

where $L$ is given by $(39)$ and [.] is the integer part function. We have the following lemma.

Lemma 3.3. Let $\delta \in(0, \rho), \varepsilon \in\left(0, \varepsilon_{0}\right)$, with $\varepsilon_{0}$ given by (45), and set

$$
r_{i}=(1-\varepsilon)^{i} \rho \sin \frac{\delta}{2 \rho},
$$


for $i \in \mathbb{N}$. Let $p$ and $q$ be any two points in a connected component of $S_{m}^{\delta}$. Then there exist an integer $N \leq N_{0}$, with $N_{0}$ given by (46), and a sequence of points $\left\{p_{1}, \ldots, p_{N}\right\}$ in $S_{m}^{\delta / 2}$ such that

$$
\begin{aligned}
& p, q \in \bigcup_{i=0}^{n} \overline{\mathcal{U}}_{r_{i} / 4}\left(p_{i}\right) ; \\
& \mathcal{U}_{r_{0}}\left(p_{i}\right) \subset S_{m}, \quad i=0, \ldots, N, \\
& p_{i+1} \in \overline{\mathcal{U}_{r_{i} / 4}\left(p_{i}\right)}, \quad i=0, \ldots, N-1,
\end{aligned}
$$

where $\mathcal{U}_{r_{i}}\left(p_{i}\right)$ are defined as in (5).

Proof. Let $\gamma$ be a path as in Lemma 3.2 and denote by $s$ its arclength. Set $p_{0}=p$ and define $p_{i}=\gamma\left(r_{i} / 4\right)$, for each $i=1, \ldots, N-1$, and $p_{N}=q$. Here, $N$ is the largest integer such that

$$
\sum_{i=0}^{N-1} \frac{r_{i}}{4} \leq L .
$$

Since $\varepsilon<\varepsilon_{0}$, we have

$$
\sum_{i=0}^{N_{0}-1} \frac{r_{i}}{4}>2 L,
$$

and hence such $N$ exists and we can assume that $N \leq N_{0}$, where $N_{0}$ is defined by (46). Since $\gamma \subset S_{m}^{\delta / 2}$, the assertion of the theorem easily follows from 100 .

For a fixed direction $\ell \in \mathbb{S}^{n}$, we denote by $\ell^{\perp}$ the orthogonal subspace to $\ell$, i.e.

$$
\ell^{\perp}=\left\{z \in \mathbb{R}^{n+1}: z \cdot \omega=0\right\} .
$$

Lemma 3.4. Let $p \in S$ and $u: B_{r} \cap T_{p} S \rightarrow \mathbb{R}$ be a $C^{2}$ map as in (5), with $r<\rho$. Let $\ell \in \mathbb{S}^{n}$ be such that

$$
\nu_{p} \cdot \ell>0 \text { and }\left|\ell-\nu_{p}\right|<\varepsilon,
$$

for some $0 \leq \varepsilon<1$. There exists a $C^{2}$ function $v: B_{r \sqrt{1-\varepsilon^{2}}} \cap \ell^{\perp} \rightarrow \mathbb{R}$ such that the set

$$
V=\left\{p+y+v(y) \ell: y \in B_{r \sqrt{1-\varepsilon^{2}}} \cap \ell^{\perp}\right\}
$$

is contained in $\mathcal{U}_{r}(p)$. Moreover, the estimate

$$
\|v\|_{\infty} \leq\|u\|_{\infty}+\sqrt{2} \varepsilon r
$$

holds.

Proof. Let $q=p+x+u(x) \nu_{p}$ be a point in $\mathcal{U}_{r}(p)$, with

$$
|x|<r \sqrt{1-\varepsilon^{2}} \text {. }
$$

By the implicit function theorem, if $\nu_{q} \cdot \ell>0$, then $S$ can be locally represented as a graph of function near $q$ over the hyperplane $\ell^{\perp}$. Let $A \in \mathrm{SO}(n+1)$ be a special orthogonal matrix such that

$$
A \nu_{p}=\ell
$$

and let $y \in \ell^{\perp}$ be such that

$$
y=A x .
$$

Since $A \in \mathrm{SO}(n+1)$ we have $|x|=|y|$ and then

$$
|y|<r \sqrt{1-\varepsilon^{2}} .
$$


From triangle and Cauchy-Schwarz inequalities we have that

$$
\nu_{q} \cdot \ell \geq \nu_{q} \cdot \nu_{p}-\left|\ell-\nu_{p}\right|
$$

(9) and (51) yield that

$$
\nu_{q} \cdot \ell \geq \sqrt{1-\frac{|x|^{2}}{\rho^{2}}}-\varepsilon,
$$

which implies that $\nu_{q} \cdot \ell>0$ on account of (54). Therefore any point $q \in V$ can be written both as $q=p+x+u(x) \nu_{p}$ and as $q=p+y+v(y) \ell$ for some $x \in T_{p} S$ and $y \in \ell^{\perp}$. In particular

$$
y+v(y) \ell=x+u(x) \nu_{p}
$$

and, since $y=A x$, we have

$$
(I-A) x+u(x) \nu_{p}=v(y) \ell .
$$

By taking the scalar product with $\ell$, we readily obtain

$$
|v(\xi)| \leq|I-A||x|+|u(x)| .
$$

The matrix $A$ can be choosen such that

$$
|I-A| \leq 2 \sqrt{1-\ell \cdot \nu_{p}} \leq \sqrt{2} \varepsilon,
$$

and (55) implies the last part of the statement.

It will be important to compare the normal vectors to two surfaces which are graphs of function over the same domain. We have the following Lemma.

Lemma 3.5. Let $u_{1}, u_{2} \in C^{1}\left(B_{r} \cap e_{n+1}^{\perp}\right)$ and assume that

$$
\left|\nabla u_{2}\left(x_{0}\right)-\nabla u_{1}\left(x_{0}\right)\right|<\varepsilon,
$$

for some $x_{0} \in B_{r} \cap e_{n+1}^{\perp}$. Let $p_{i}=x_{0}+u_{i}\left(x_{0}\right) e_{n+1}, i=1,2$. Then

$$
\left|\nu_{p_{1}}-\nu_{p_{2}}\right| \leq \frac{\sqrt{5}}{2} \varepsilon
$$

where

$$
\nu_{p_{i}}=\frac{-\nabla u_{i}\left(x_{0}\right)+e_{n+1}}{\sqrt{1+\left|\nabla u_{i}\left(x_{0}\right)\right|^{2}}}
$$

is the inward normal to the graph of $u_{i}$ at $p_{i}, i=1,2$.

Proof. Since the eigenvalues of the Hessian of the function $x \mapsto \sqrt{1+|x|^{2}}$ are uniformly bounded by 1 , then its gradient is Lipschitz continuous with constant 1 and we have that

$$
\left|\frac{\nabla u_{1}(x)}{\sqrt{1+\left|\nabla u_{1}(x)\right|^{2}}}-\frac{\nabla u_{2}(x)}{\sqrt{1+\left|\nabla u_{2}(x)\right|^{2}}}\right| \leq\left|\nabla u_{1}(x)-\nabla u_{2}(x)\right|
$$

Moreover, we have that

$$
\left|\frac{1}{\sqrt{1+\left|\nabla u_{1}(x)\right|^{2}}}-\frac{1}{\sqrt{1+\left|\nabla u_{2}(x)\right|^{2}}}\right| \leq \frac{1}{2}|| \nabla u_{1}(x)|-| \nabla u_{2}(x)|| .
$$

From triangle inequality and from $(57)$ and $(58)$ we readily obtain $(56)$. 


\section{Proof of Theorem 1.1}

The proof of Theorem 1.1 relies upon a quantitative study of the method of moving planes and it consists of several steps, as we sketch in the following.

Step 1. We fix a direction $\omega$, apply the method of moving planes, and find a critical position which defines a critical hyperplane $\pi_{m}$, as described in Subsection 2.2. By using the smallness of $\operatorname{osc}(H)$, we can prove that (up to a connected component) the surface $S$ and the reflected cap $S_{m}$ are close. Hence, the union of the cap and the reflected cap provides a symmetric set in the direction $\omega$ which gives information about the approximate symmetry of $S$ in the direction $\omega$. It is important to notice that the estimates do not depend on the chosen direction.

Step 2. We apply Step 1 in $n+1$ orthogonal directions and we obtain a point $\mathcal{O}$ as the intersection of the corresponding $n+1$ critical hyperplanes. Since the estimates in Step 1 do not depend on the direction, the point $\mathcal{O}$ can be chosen as an approximate center of symmetry. Moreover, any critical hyperplane in any other direction is far from $\mathcal{O}$ less than some constant times osc $(H)$.

Step 3. Again by using the estimates in Step 1, we can define two balls centered at $\mathcal{O}$ such that estimate (3) holds.

We notice that once we have the approximate symmetry in one direction, i.e. Step 1, then the argument for proving Steps 2 and 3 is well-established (see [1, Section 4]). In the following we will prove Step 1, which is our main result of this section and, for the sake of completeness, we give a sketch of the proof for Steps 2 and 3 .

4.1. Step 1. Approximate symmetry in one direction. We apply the moving plane procedure as described in Subsection 2.2. Let $\omega \in \mathbb{S}^{n}$ be a direction in $\mathbb{R}^{n+1}$ and let $S_{m}, \hat{S}_{m}$ be defined as in 29$)$. Let

$p_{0}$ be a tangency point between $S_{m}$ and $\hat{S}_{m}$,

and denote by $\Sigma$ and $\hat{\Sigma}$ the connected components of $S_{m}$ and $\hat{S}_{m}$, respectively, containing $p_{0}$ or having $p_{0}$ on their boundary. Let $S^{*}$ be the reflection of $S$ about $\pi_{m}$. For a point $p$ in $S$ (or $S^{*}$ ), we denote by $\nu_{p}$ the normal vector to $S$ (or to $S^{*}$ ) at $p$. We will use this notation when it does not create ambiguity: the choice of the vector normal and of the surface is implied by the point itself. When $p \in S \cap S^{*}$ is a point of tangency between $S$ and $S^{*}$, then the normal vector at $p$ is the same for both the surfaces, and the notation is coherent. When this notation creates an ambiguity, i.e. for nontangency points in $S \cap S^{*}$, we will specify the dependency on the surface. For points on $\partial \Sigma$ (or $\partial \hat{\Sigma}$ ) we will denote by $\nu$ the Gauss map on $\partial \Sigma$ (or $\partial \hat{\Sigma}$ ) which is induced by the one on $S^{*}$ (or $S$ ).

The main goal of Step 1 is to prove the following result of approximate symmetry in one direction.

Theorem 4.1. There exists a positive constant $\varepsilon$ such that if

$$
\operatorname{osc}(H) \leq \varepsilon,
$$

then for any $p \in \Sigma$ there exists $\hat{p} \in \hat{\Sigma}$ such that

$$
|p-\hat{p}|+\left|\nu_{p}-\nu_{\hat{p}}\right| \leq C \operatorname{osc}(H) .
$$

Here, the constants $\varepsilon$ and $C$ depend only on $n, \rho,|S|$ and do not depend on the direction $\omega$.

Before giving the proof of Theorem 4.1 , we provide two preliminary results about the geometry of $\Sigma$. For $t>0$ we set

$$
\Sigma^{t}=\left\{p \in \Sigma: d_{\Sigma}(p, \partial \Sigma)>t\right\}
$$


The following two lemmas show some conditions implying that $\Sigma^{t}$ is connected for $t$ small enough.

Lemma 4.2. Assume that there exists $\mu \leq \frac{1}{2}$ such that

$$
\nu_{p} \cdot \omega \leq \mu
$$

for every $p$ on the boundary of $\Sigma$. Then $\Sigma^{t}$ is connected for any $0<t \leq t_{0}$, where

$$
t_{0}=\frac{\rho}{2 \sqrt{n}} \sqrt{1-2 \mu^{2}} .
$$

Proof. Let $S^{*}$ be the reflection of $S$ about $\pi_{m}$. We notice that, by construction of the method of moving planes, $\Sigma$ and $\pi_{m}$ enclose a bounded simply connected domain of $\mathbb{R}^{n+1}$. Moreover, $\nu_{p} \cdot \omega \geq 0$ on $\partial \Sigma$ and equation (60) implies that $\pi_{m}$ intersects $S^{*}$ transversally. Hence, the boundary of $\Sigma$ is a manifold of class $C^{2}$. We prove that the boundary of $\Sigma^{t}$ lies in a tubular neighbourhood of the boundary of $\Sigma$ in $S^{*}$. Then, since $\Sigma$ is connected, every two points in $\Sigma^{t}$ can be joined by a curve in $\Sigma$ which can be pushed into $\Sigma^{t}$ by using the normal vector field to the boundary $\Sigma$.

According to Section 2.3 , we denote the boundary of $\Sigma$ by $\Sigma^{\prime}$ and we orient $\Sigma^{\prime}$ by the Gauss map satisfying

$$
\nu_{p} \cdot \nu_{p}^{\prime}=1-\left(\nu_{p} \cdot \omega\right)^{2}
$$

(see formula $(32)$ ). Hence, from $(60)$, we have that

$$
\nu_{p} \cdot \nu_{p}^{\prime} \geq 1-\mu^{2}
$$

Since the principal curvatures of $S$ are bounded by $\rho^{-1}$, from Proposition 2.7 the principal curvatures $\kappa_{i}^{\prime}$ of $\Sigma^{\prime}$ satisfy

$$
\left|\kappa_{i}^{\prime}\right| \leq \frac{1}{\rho\left(1-\mu^{2}\right)}, \quad i=1, \ldots, n-1 .
$$

From Lemma 3.4, we can write $S^{*}$ as a graph of function $u: B_{r} \cap\left(\nu_{p}^{\prime}\right)^{\perp} \rightarrow \mathbb{R}$, with $r=\rho \sqrt{1-2 \mu^{2}}$. Moreover, (61) and Lemma 2.1 yield that $\Sigma^{\prime}$ is locally the graph of $u$ restricted to $B_{r} \cap T_{p} \Sigma^{\prime}$. Taking into account that $\left(\nu_{p}^{\prime}\right)^{\perp}=T_{p} \Sigma^{\prime} \oplus\langle\omega\rangle$, we consider the subset of $S^{*}$ given by

$$
Q(p)=\left\{q=p+\xi+s \omega+u(\xi+s \omega) \nu_{p}^{\prime}: \quad \xi \in B_{r} \cap T_{p} \Sigma^{\prime},|s| \leq t_{0}\right\},
$$

which contains a tubular neighborhood of $\Sigma^{\prime} \cap B_{t_{0}}(p)$ of radius at least $t_{0}$. Hence, the set

$$
\mathcal{Q}=\bigcup_{p \in \Sigma^{\prime}} Q(p)
$$

contains a tubular neighborhood of $\Sigma^{\prime}$ in $S^{*}$ of radius at least $t_{0}$ and we conclude.

Lemma 4.3. Let $0<\delta \leq \rho(8 \sqrt{n})^{-1}$. If we suppose that there exists a connected component $\Gamma^{\delta}$ of $\Sigma^{\delta}$ satisfying

$$
0 \leq \nu_{p} \cdot \omega \leq \frac{1}{8}
$$

then, $\Sigma^{\delta}$ is connected.

Proof. In order to simplify the notation we let $\mu_{0}=1 / 8$. Notice that the interior and exterior touching balls at every boundary points of $\Gamma^{\delta}$ intersects $\pi_{m}$. By using this argument and after elementary but tedious calculations, we can prove that any $q \in \Sigma \backslash \Gamma^{\delta}$ is such that

$$
d_{\Sigma}\left(q, \Gamma^{\delta}\right) \leq \rho \arcsin \left(\left(1+2 \mu_{0}\right) \frac{\delta}{\rho}\right) .
$$


In particular, for any $q \in \partial \Sigma$ there exists $p \in \partial \Sigma^{\delta}$ such that

$$
d_{\Sigma}(q, p) \leq \rho \arcsin \left(\left(1+2 \mu_{0}\right) \frac{\delta}{\rho}\right),
$$

and from Lemma 2.1 we obtain that

$$
\left|\nu_{p}-\nu_{q}\right| \leq \sqrt{2} \arcsin \left(\left(1+2 \mu_{0}\right) \frac{\delta}{\rho}\right) .
$$

By writing $\nu_{q} \cdot \omega=\nu_{p} \cdot \omega-\left(\nu_{q}-\nu_{p}\right) \cdot \omega$ and by triangle inequality we get

$$
\left|\nu_{q} \cdot \omega\right| \leq \mu_{0}+\sqrt{2} \arcsin \left(\left(1+2 \mu_{0}\right) \frac{\delta}{\rho}\right) ;
$$

our assumptions on $\delta$ implies the following (rougher but simpler) bound:

$$
\left|\nu_{q} \cdot \omega\right| \leq 2 \mu_{0}+\frac{1}{2}
$$

Now we use Lemma 4.2 by setting $\mu=2 \mu_{0}+1 / 2$ and imposing that $\delta \leq t_{0}$, and we conclude.

Now, we focus on the proof of Theorem 4.1. It will be divided in four cases, which we study in the following subsections. In each case, $\delta$ will be fixed to be

$$
\delta=\min \left(\frac{\rho}{2^{6}}, \frac{\rho}{8 \sqrt{n}}\right) .
$$

Moreover, the constants $\varepsilon$ and $C$ can be chosen as

$$
\varepsilon=\min \left\{\varepsilon_{0}, \varepsilon_{1}, \varepsilon_{2}, \varepsilon_{3}\right\},
$$

and

$$
C=\frac{5}{4} C_{1} K_{1} K_{2} K_{3}
$$

respectively. Here, $\varepsilon_{0}$ is given by (45), and $\varepsilon_{1}, \varepsilon_{2}, \varepsilon_{3}$ and $C_{1}$ will be defined in the following. Moreover, $K_{1}, K_{2}, K_{3}$ are given by Lemmas 2.3, 2.4, 2.6, respectively, where $M$ is chosen accordingly to Lemma 2.1 by assuming that $|x| \leq \rho / 2$. Hence, the constants $\varepsilon$ and $C$ depend only on $n$ and upper bounds on $\rho^{-1}$ and $|S|$.

4.1.1. Case 1. $d_{\Sigma}\left(p_{0}, \partial \Sigma\right)>\delta$ and $d_{\Sigma}(p, \partial \Sigma) \geq \delta$. In this first case we assume that $p_{0}$ and $p$ are interior points of $\Sigma$, which are far from $\partial \Sigma$ more than $\delta$. We remark that in this case, $p_{0}$ is an interior touching point between $\Sigma$ and $\hat{\Sigma}$, so that case (i) in the method of moving planes occurs. We first assume that $p_{0}$ and $p$ are in the same connected component of $\Sigma^{\delta}$; then, Lemma 4.3 will be used in order to show that $\Sigma^{\delta}$ is in fact connected.

Let

$$
r_{0}=\rho \sin \frac{\delta}{2 \rho}
$$

Since $p$ and $p_{0}$ are in a connected component of $\Sigma^{\delta}$, there exist: $\left\{p_{1}, \ldots, p_{N}\right\}$ in the connected component of $\Sigma^{\delta / 2}$ containing $p_{0}$, a chain $\left\{\mathcal{U}_{r_{0}}\left(p_{i}\right)\right\}_{\{i=0, \ldots, N\}}$ of open sets of $\Sigma$ and a sequence of maps $u_{i}: B_{r_{0}} \cap T_{p_{i}} \Sigma \rightarrow \mathbb{R}, i=0, \ldots, N$, as in Lemma 3.3, where $r_{i}=(1-\varepsilon)^{i} r_{0}$. We notice that $\Sigma$ and $\hat{\Sigma}$ are tangent at $p_{0}$ and that in particular the two normal vectors to $\Sigma$ and $\hat{\Sigma}$ at $p_{0}$ coincide. We stress that $\hat{\Sigma} \subset S$ and that, since $r_{0}<\rho$, from Lemma 2.1 we have that $S$ is locally represented near $p_{0}$ as a graph of a map $\hat{u}_{0}: B_{r_{0}} \cap T_{p_{0}} S \rightarrow \mathbb{R}$. 
Lemma 2.1 implies that $\left|\nabla u_{0}\right|,\left|\nabla \hat{u}_{0}\right| \leq M$ in $B_{r_{0}} \cap T_{p_{0}} \Sigma$, where $M$ is some constant which depends only on $r_{0}$, i.e. only on $\rho$. Now, we use Lemma 2.3 since $u_{0}(0)=\hat{u}_{0}(0)$ and $u_{0} \geq \hat{u}_{0}$, (18) gives

$$
\left\|u_{0}-\hat{u}_{0}\right\|_{C^{1}\left(B_{r_{0} / 4} \cap T_{p_{0}} \Sigma\right)} \leq K_{1} \operatorname{osc}(H),
$$

where $K_{1}$ depends only on $n$ and $M$. We notice that from w0 wave that $p_{1} \in \overline{\mathcal{U}}_{r_{0} / 4}\left(p_{0}\right)$. Let $x_{1}$ be the projection of $p_{1}$ onto $T_{p_{0}} \Sigma$ and let

$$
\hat{p}_{1}:=p_{0}+x_{1}+\hat{u}_{0}\left(x_{1}\right) \nu_{p_{0}} \in \hat{\Sigma} .
$$

From 62 we obtain that

$$
\left|\nabla u_{0}\left(x_{1}\right)-\nabla \hat{u}_{0}\left(x_{1}\right)\right| \leq K_{1} \operatorname{osc}(H),
$$

and therefore Lemma 3.5 yields

$$
\left|\nu_{p_{1}}-\nu_{\hat{p}_{1}}\right| \leq \frac{\sqrt{5}}{2} K_{1} \operatorname{osc}(H)
$$

As already mentioned, we have a local parametrization of $\Sigma$ in a neighborhood of $p_{1}$ as a graph of the $C^{2}$ function $u_{1}: B_{r_{0}} \cap T_{p_{1}} \Sigma \rightarrow \mathbb{R}$. Lemma 3.4 and $(63)$ imply that $S$ can be locally parameterized by a graph of function $\hat{u}_{1}: B_{r_{1}} \cap T_{p_{1}} \Sigma \rightarrow \mathbb{R}$, being $r_{1}<r_{0} \sqrt{1-\frac{5}{4} K_{1}^{2} \varepsilon^{2}}$, since $\varepsilon \leq \varepsilon_{1}$ with

$$
\varepsilon_{1}=\left(1+\frac{5}{4} K_{1}^{2}\right)^{-1}
$$

Moreover, (53) yields that

$$
\left|u_{1}(0)-\hat{u}_{1}(0)\right| \leq\left\|u_{0}-\hat{u}_{0}\right\|_{C^{0}\left(B_{r_{0} / 4} \cap T_{p_{0}} \Sigma\right)}+\sqrt{5} r_{0} K_{1} \operatorname{osc}(H) ;
$$

from (62) and since $u_{1}-\hat{u}_{1} \geq 0$ by construction, we find that

$$
0 \leq u_{1}(0)-\hat{u}_{1}(0) \leq\left(1+r_{0} \sqrt{5}\right) K_{1} \operatorname{osc}(H) .
$$

We use Lemma 2.3 and obtain that

$$
\left\|u_{1}-\hat{u}_{1}\right\|_{C^{1}\left(B_{r_{1} / 4} \cap T_{p_{1}} \Sigma\right)} \leq K_{1}\left[\left(1+r_{0} \sqrt{5}\right) K_{1}+1\right] \operatorname{osc}(H) .
$$

Now, (65) is the analogue of 62 with $p_{1}$ instead of $p_{0}$, and we can iterate until we obtain two functions

$$
u_{N}, \hat{u}_{N}: B_{r_{N}} \cap T_{p} \Sigma \rightarrow \mathbb{R}
$$

such that

$$
\left\|u_{N}-\hat{u}_{N}\right\|_{C^{1}\left(B_{r_{N} / 4} \cap T_{p} \Sigma\right)} \leq C_{1} \operatorname{osc}(H) .
$$

A choice of $\hat{p}$ as in the statement of Theorem 4.1 is then given by

$$
\hat{p}=p+\hat{u}_{N}(0) \nu_{p}
$$

since 59 is implied by (66) and Lemma 3.5 .

We notice that a choice of the constant $C_{1}$ in $(66)$ is given by

$$
C_{1}=\left(\left(1+r_{0} \sqrt{5}\right) K_{1}+1\right)^{N_{0}+1},
$$

where $N_{0}$ is given by (46). Hence the constant $C_{1}$ depends only on $n, \delta / \rho$, and an upper bound on $|S|$. 
Once we have (66) for any $p$ in a connected component of $\Sigma^{\delta}$, we have in fact that

$$
\nu_{q} \cdot \omega \leq \frac{1}{8}
$$

for any point $q$ at the boundary of such a connected component, as it is implied by the following Lemma.

Lemma 4.4. Let $q \in \Sigma$ be such that $d_{\Sigma}(q, \partial \Sigma) \leq \delta$. Assume that the point

$$
\hat{q}=q-\alpha \nu_{q}
$$

is on $\hat{\Sigma}$ and is such that

$$
\left|\nu_{q}-\nu_{\hat{q}}\right| \leq \alpha
$$

with $\alpha+2 \delta<\rho$. Then we have that

$$
0 \leq \nu_{q} \cdot \omega \leq \sqrt{8 \frac{\delta^{2}}{\rho^{2}}+\frac{\alpha}{2}} .
$$

Proof. Let $q^{m}$ be the reflection of $q$ about $\pi_{m}$ and let

$$
t=\nu_{q} \cdot \omega
$$

By construction of the method of moving planes, it is clear that $t \geq 0$ and the first inequality in 69 follows. We denote by $\nu_{q^{m}}$ the inner normal vector to $S$ at $q^{m}$. Since $\nu_{q} \cdot \omega=-\nu_{q^{m}} \cdot \omega$ and $\nu_{q}-\nu_{q^{m}}=2 t \omega$, we have that

$$
\nu_{q} \cdot \nu_{q^{m}}=1-2 t^{2} .
$$

We notice that $q^{m}$ and $\hat{q}$ both lie in $S$ and $\left|q^{m}-\hat{q}\right| \leq \alpha+2 \delta$, which implies that $\hat{q} \in \mathcal{U}_{\rho}\left(q^{m}\right)$ provided that $\alpha+2 \delta<\rho$. Hence, $(9)$ yields that

$$
\nu_{\hat{q}} \cdot \nu_{q^{m}} \geq \sqrt{1-\left(\frac{\alpha+2 \delta}{\rho}\right)^{2}} .
$$

From $(68)$ and $(70)$ we find that

$$
1-2 t^{2} \geq \sqrt{1-\left(\frac{\alpha+2 \delta}{\rho}\right)^{2}}-\alpha,
$$

which gives

$$
t^{2} \leq \frac{1}{2}\left(\frac{\alpha+2 \delta}{\rho}\right)^{2}+\frac{\alpha}{2}
$$

and we obtain the second inequality in 69 .

The conclusion of Case 1 follows from the following argument. From (66) we know that for any $q$ on the boundary of the connected component of $\Sigma^{\delta}$ containing $p_{0}$ there exists $\hat{q} \in \hat{\Sigma}$ such that

$$
|q-\hat{q}|+\left|\nu_{q}-\nu_{\hat{q}}\right| \leq C_{1} \operatorname{osc}(H) .
$$

We apply Lemma 4.4 by letting $\alpha=C_{1} \operatorname{osc}(H)$ and, since $\varepsilon \leq \varepsilon_{2}$, with

$$
\varepsilon_{2} \leq \frac{1}{2^{6} C_{1}}
$$

we obtain that $0 \leq \nu_{q} \cdot \omega \leq 1 / 8$. Hence, from Lemma 4.3 we have that $\Sigma^{\delta}$ is connected and we conclude. 
4.1.2. Case 2: $d_{\Sigma}\left(p_{0}, \partial \Sigma\right) \geq \delta$ and $d_{\Sigma}(p, \partial \Sigma)<\delta$. Here the idea consists in extending the estimate in Subsection 4.1.1 to the whole $\Sigma$. This will be done by using Carleson type estimates given by Lemma 2.4. We remark that its application is not trivial, since we need more information on how $S$ intersects $\pi_{m}$.

Accordingly to $(29)$ in Subsection 2.2, for a given point $p \in \Sigma$ such that $d_{\Sigma}(p, \partial \Sigma) \leq \delta$, we denote by $p^{m}$ the point of $S$ obtained by reflecting $p$ about $\pi_{m} . S$ can be locally written as a graph of function $u: B_{\rho} \cap T_{p} S \rightarrow \mathbb{R}$. For $0<r<\rho$, we define $U_{r}^{*}(p)$ as the reflection of $\mathcal{U}_{r}\left(p^{m}\right)$ about $\pi_{m}$ and we denote by $U_{r}(p)$ the subset of $\Sigma$ obtained by

$$
U_{r}(p)=U_{r}^{*}(p) \cap\left\{q \in \mathbb{R}^{n+1}: q \cdot \omega<m\right\} .
$$

Moreover, we denote by $E_{r}$ the open subset of $B_{r} \cap T_{p} \Sigma$ such that

$$
U_{r}(p)=\left\{p+x+u(x) \nu_{p}: x \in E_{r}\right\} .
$$

The next result is a consequence of Propositions 2.7, 2.8 in Subsection 2.3.

Lemma 4.5. Let $q \in \Sigma$ be such that $d_{\Sigma}(q, \partial \Sigma)=\delta$ and $0 \leq \nu_{q} \cdot \omega \leq 1 / 4$. Let $U^{\prime}=U_{\sqrt{2} \rho / 8}^{*}(q) \cap \pi_{m}$ and $U^{\prime \prime}$ be the orthogonal projection of $U^{\prime}$ onto $T_{q} \Sigma$. Then $U^{\prime \prime}$ is a hypersurface of class $C^{2}$ of $T_{q} \Sigma$ whose principal curvatures are bounded by

$$
\mathcal{K}=\frac{4 \delta}{\rho^{2}} .
$$

Proof. We notice that since $d_{\Sigma}(q, \partial \Sigma)=\delta$ then $U^{\prime} \neq \emptyset$. Let $\zeta \in U^{\prime}$ be arbitrary. Since the projection $\operatorname{pr}(\zeta)$ of $\zeta$ on $T_{q} \Sigma$ is in $\bar{B}_{\sqrt{2} \rho / 8}$, from (9) in Lemma 2.1 we know that

$$
\left|\nu_{q}-\nu_{\zeta}\right| \leq \frac{1}{4}
$$

Since $\nu_{\zeta} \cdot \omega=\nu_{q} \cdot \omega+\left(\nu_{\zeta}-\nu_{q}\right) \cdot \omega$, we have that

$$
\left|\nu_{\zeta} \cdot \omega\right| \leq \frac{1}{2}
$$

which implies that $\pi_{m}$ intersects $U_{\sqrt{2} \rho / 8}^{*}(q)$ transversally, and so $U^{\prime \prime}$ is a hypersurface of $T_{q} \Sigma$. Since the principal curvatures of $S$ are bounded by $1 / \rho, 35$ implies that the principal curvatures of $U^{\prime \prime}$ satisfy

$$
\left|\kappa_{i}^{\prime \prime}(\operatorname{pr}(\zeta))\right| \leq \frac{1}{\rho\left|\nu_{\zeta} \cdot \nu_{\zeta}^{\prime}\right|} \cdot \frac{\omega \cdot \nu_{q}}{\left[\left(\omega \cdot \nu_{q}\right)^{2}+\left(\nu_{q} \cdot \nu_{\zeta}^{\prime}\right)^{2}\right]^{3 / 2}}, \quad i=1, \ldots, n-1,
$$

where $\nu^{\prime}$ is the Gauss map of $U^{\prime}$ viewed as a hypersurface of $\pi_{m}$ satisfying

$$
\nu_{\zeta} \cdot \nu_{\zeta}^{\prime}=1-\left(\nu_{\zeta} \cdot \omega\right)^{2} .
$$

Hence,

$$
\left|\kappa_{i}^{\prime \prime}(\operatorname{pr}(\zeta))\right| \leq \frac{\omega \cdot \nu_{q}}{\rho\left|\nu_{\zeta} \cdot \nu_{\zeta}^{\prime}\right|\left|\nu_{q} \cdot \nu_{\zeta}^{\prime}\right|^{3}}, \quad i=1, \ldots, n-1 .
$$

From 73 and $(74)$, we obtain that

$$
\nu_{\zeta} \cdot \nu_{\zeta}^{\prime} \geq \frac{3}{4}
$$

By writing

$$
\nu_{q} \cdot \nu_{\zeta}^{\prime}=\left(\nu_{q}-\nu_{\zeta}\right) \cdot \nu_{\zeta}^{\prime}+\nu_{\zeta} \cdot \nu_{\zeta}^{\prime}
$$


and using $(72)$ and $(76)$ we get

and from 75 and 76 we conclude.

$$
\nu_{q} \cdot \nu_{\zeta}^{\prime} \geq \frac{1}{2}
$$

In the next lemma we give a bound which will be useful in the sequel.

Lemma 4.6. Let $q$ and $\alpha$ be as in Lemma 4.4. Then, we have that

$$
0 \leq \nu_{\zeta} \cdot \omega \leq \sqrt{8 \frac{\delta^{2}}{\rho^{2}}+\frac{\alpha}{2}}+\frac{\sqrt{2}}{\rho} d_{\Sigma}(q, \zeta),
$$

for any $\zeta \in \bar{U}_{\rho}(q)$, where $U_{\rho}(q)$ is defined as in 71 ).

Proof. Let $\zeta \in \bar{U}_{\rho}(q)$. By construction we have that $\nu_{\zeta} \cdot \omega \geq 0$. Since

$$
\nu_{\zeta} \cdot \omega \leq \nu_{q} \cdot \omega+\left|\nu_{\zeta}-\nu_{q}\right|
$$

from (9) and (69) we conclude.

Now we are ready to prove Theorem 4.1 for Case 2. Let

$$
\varepsilon_{3}=\frac{\delta}{\rho C_{1}}
$$

where $C_{1}$ is given by (67). We assume that $d_{\Sigma}\left(p_{0}, \partial \Sigma\right) \geq \delta$ and $d_{\Sigma}(p, \partial \Sigma)<\delta$. By arguing as in Case 1, we have that $\Sigma^{\delta}$ is connected. Let $q \in \Sigma$ and $\bar{p} \in \partial \Sigma$ be such that

$$
d_{\Sigma}(p, q)+d_{\Sigma}(p, \partial \Sigma)=\delta,
$$

and

$$
d_{\Sigma}(p, \bar{p})=d_{\Sigma}(p, \partial \Sigma)
$$

(we notice that our choice of $\delta$ implies that $q$ and $\bar{p}$ exist).

Since $d_{\Sigma}(q, \partial \Sigma)=\delta$, from Case 1 we have that there exists $\hat{q} \in \hat{\Sigma}$ such that

$$
|q-\hat{q}|+\left|\nu_{q}-\nu_{\hat{q}}\right| \leq C_{1} \operatorname{osc}(H)
$$

(see formula (66)). From the proof of Case 1, it is clear that $\hat{q}$ can be chosen as

$$
\hat{q}=q-\alpha \nu_{q}
$$

for some $0 \leq \alpha \leq C_{1} \operatorname{osc}(H)$. Let

$$
r=\frac{\rho}{8}
$$

We define the sets $U_{r}(q) \subset \Sigma, E_{r} \subseteq B_{r} \cap T_{q} \Sigma$, and the map $u: E_{r} \rightarrow \mathbb{R}$ as in 717 with $q$ in place of $p$. Since $\hat{q} \in \hat{\Sigma} \subset S$ and $\left|\nu_{q}-\nu_{\hat{q}}\right| \leq C_{1} \operatorname{osc}(H)$, from Lemma 3.4 we have that $S$ can be locally written (around $\hat{q}$ ) as a graph of function $\hat{u}$ over $T_{q} \Sigma \cap B{ }_{\rho \sqrt{1-C_{1}^{2} \varepsilon_{3}^{2}}}$ and in particular over $T_{q} \Sigma \cap B_{r}$ (which is justified by our choice of $\varepsilon_{3}$ ).

We notice that Lemma 2.2 implies that $p, \bar{p} \in \bar{U}_{r}(q)$. Let $\partial E_{r}$ be the boundary of $E_{r}$ in $T_{q} \Sigma$ and let $\bar{x} \in \partial E_{r}$ be the projection of $\bar{p}$. Since $d_{\Sigma}(q, \bar{p})=\delta$, from Lemma 2.2 we have that

$$
\rho \sin \frac{\delta}{\rho} \leq|\bar{x}| \leq \delta \text {. }
$$

Let $U^{\prime}=U_{r}^{*}(q) \cap \pi_{m}$ and let $U^{\prime \prime}$ be the projection of $U^{\prime}$ onto $T_{q} \Sigma$ (as in Lemma 4.5). Notice that by definition $U^{\prime \prime}$ is contained in $\partial E_{r}$ and, in particular, $u=\hat{u}$ on $U^{\prime \prime}$. From Lemma 4.4 and Lemma 4.5, we have that the principal curvatures of $U^{\prime \prime}$ are uniformly bounded by $\mathcal{K}$. We notice that our choice of $\delta$ implies that $\mathcal{K} \leq \frac{1}{16 \rho}$. 


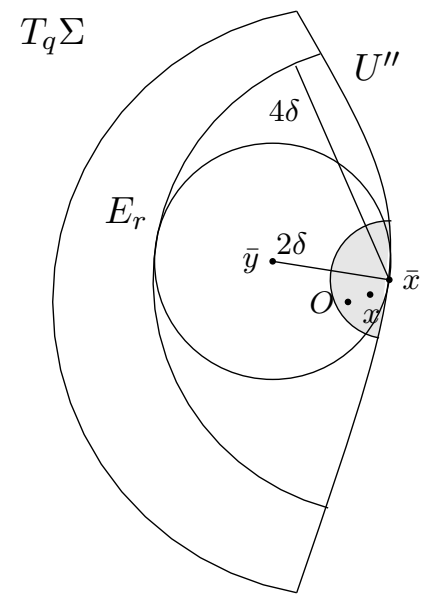

Figure 2. Case 2 in the proof of Theorem 4.1. The shadow region is the set $B_{\delta}(\bar{x}) \cap E_{r}$.

Let $x$ be the projection of $p$ over $T_{q} \Sigma$. From we have that $B_{4 \delta}(\bar{x}) \cap \partial E_{r} \subset U^{\prime \prime}$ and we can apply Lemma 2.4 and obtain that

$$
\sup _{B_{\delta}(\bar{x}) \cap E_{r}}(u-\hat{u}) \leq K_{2}((u-\hat{u})(\bar{y})+\operatorname{osc}(H)),
$$

with $\bar{y}=\bar{x}+2 \delta \nu_{\bar{x}}^{\prime \prime}$, where $\nu_{\bar{x}}^{\prime \prime}$ is the interior normal to $U^{\prime \prime}$ at $\bar{x}$ (see Figure 2). We notice that $x \in B_{\delta}(\bar{x}) \cap E_{r}$ and then from (81) we have that

$$
(u-\hat{u})(x) \leq K_{2}((u-\hat{u})(\bar{y})+\operatorname{osc}(H)) .
$$

Since $2 \delta<\mathcal{K}^{-1}$, the point $\bar{y}$ has distance $2 \delta$ from the boundary of $E_{r}$ and, from Lemma 2.2 we have that the point

$$
\bar{q}=q+\bar{y}+u(\bar{y}) \nu_{q}
$$

is such that

$$
d_{\Sigma}(\bar{q}, \partial \Sigma) \geq 2 \delta \text {. }
$$

Hence, from Case 1 (applied to $p_{0}$ and $\bar{q}$ ) we obtain the estimate

$$
(u-\hat{u})(\bar{y}) \leq C_{1} \operatorname{osc}(H),
$$

and from $(82)$ we get

$$
(u-\hat{u})(x) \leq C_{1} K_{2} \operatorname{osc}(H) .
$$

By letting $\hat{p}=q+x+\hat{u}(x) \nu_{q}$, and since $d_{\Sigma}(p, \partial \Sigma)>0$, a standard application of Lemma 2.3 and Lemma 3.5 yield the estimate

$$
|p-\hat{p}|+\left|\nu_{p}-\nu_{\hat{p}}\right| \leq \frac{\sqrt{5}}{2} C_{1} K_{1} K_{2} \operatorname{Osc}(H),
$$

and we complete the proof of Case 2 .

4.1.3. Case 3: $0<d_{\Sigma}\left(p_{0}, \partial \Sigma\right)<\delta$. Since $p_{0}$ is the tangency point, it is easy to show that the center of the interior touching sphere of radius $\rho$ to $S$ at $p_{0}$ lies in the half-space $\left\{q \in \mathbb{R}^{n+1}\right.$ : $q \cdot \omega \leq m\}$ (see for instance [20, Lemma 2.1]). From this, and being

$$
\left|p_{0} \cdot \omega-m\right| \leq d_{\Sigma}\left(p_{0}, \partial \Sigma\right) \leq \delta
$$




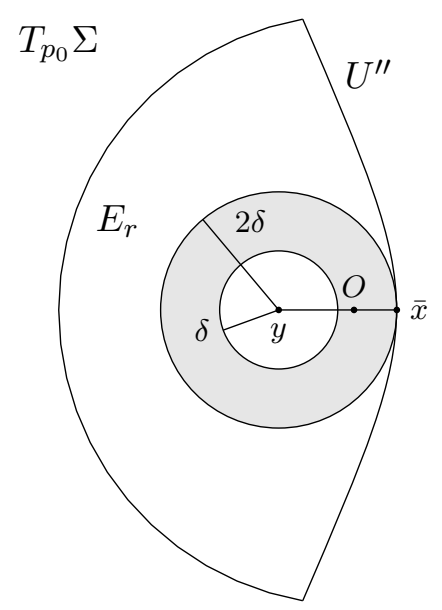

Figure 3. Case 3 in the proof of Theorem 4.1

from Lemma 4.4 (with $\alpha=0$ ) we have that

$$
\nu_{p_{0}} \cdot \omega \leq 3 \frac{\delta}{\rho}
$$

As for Case 2 (with $q$ replaced by $p_{0}$ ), we locally write $\Sigma$ and $\hat{\Sigma}$ as graphs of function $u, \hat{u}: E_{r} \rightarrow$ $\mathbb{R}$, respectively, where $E_{r} \subseteq T_{p_{0}} \Sigma$ is defined as in the introduction to this subsection, and $r$ is given by (79). Moreover, we denote by $U^{\prime \prime}$ the portion of $\partial E_{r}$ which is obtained by projecting $U_{r}^{*}\left(p_{0}\right) \cap \pi_{m}$ onto $T_{p_{0}} \Sigma$. We remark that $u=\hat{u}$ on $U^{\prime \prime}$ and that the principal curvatures of $U^{\prime \prime}$ are bounded by $\mathcal{K}$.

Let $\bar{x} \in U^{\prime \prime}$ be a point such that

$$
|\bar{x}|=\min _{x \in U^{\prime \prime}}|x| .
$$

Notice that $|\bar{x}| \leq d_{\Sigma}\left(p_{0}, \partial \Sigma\right)<\delta$. Let $\nu_{\bar{x}}^{\prime \prime}$ be the interior normal to $U^{\prime \prime}$ at $\bar{x}$, and set

$$
y=\bar{x}+2 \delta \nu_{\bar{x}}^{\prime \prime}
$$

(see Figure 3). We notice that the principal curvatures of $U^{\prime \prime}$ are bounded by $\mathcal{K}$ and $2 \delta \leq \mathcal{K}^{-1}$ and the ball $B_{2 \delta}(y) \cap T_{p_{0}} \Sigma$ is contained in $E_{r}$ and tangent to $U^{\prime \prime}$ at $\bar{x}$, with $\nu_{\bar{x}}^{\prime \prime}=-\bar{x} /|\bar{x}|$. Hence, the origin $O$ of $T_{p_{0}} \Sigma$ (i.e. the projection of $p_{0}$ over $T_{p_{0}} \Sigma$ ) lies in the annulus $\left(B_{2 \delta}(y) \backslash B_{\delta}(y)\right) \cap T_{p_{0}} \Sigma$. Hence, we can apply (27) in Lemma 2.6 (there we set: $x_{0}=\bar{x}, c=y$ and $r=2 \delta$ ) and, since $u(0)=\hat{u}(0)$, we find that

$$
\|u-\hat{u}\|_{C^{1}\left(B_{\delta / 2}(y) \cap T_{p_{0}} \Sigma\right)} \leq K_{3} \operatorname{Osc}(H)
$$

Let

$$
q=p_{0}+y+u(y) \nu_{p_{0}}, \quad \text { and } \hat{q}=p_{0}+y+\hat{u}(y) \nu_{p_{0}} .
$$

We notice that from 83 and Lemma 3.5 we have that

$$
|q-\hat{q}|+\left|\nu_{q}-\nu_{\hat{q}}\right| \leq \frac{\sqrt{5}}{2} K_{3} \operatorname{osc}(H) .
$$

Since $y$ has distance $2 \delta$ from $\partial E_{r}$, then $d_{\Sigma}(q, \partial \Sigma) \geq 2 \delta$, and we can apply Cases 1 and 2 to conclude. 
4.1.4. Case 4: $p_{0} \in \partial \Sigma$. This case is the limiting case of Case 3 for $d_{\Sigma}\left(p_{0}, \partial \Sigma\right) \rightarrow 0$. Indeed, in this case we can write $\Sigma$ and $\hat{\Sigma}$ as graphs of functions over a half-ball on $T_{p_{0}} \Sigma$. Hence the argument used in Case 3 can be adapted easily by using (28) instead of (27).

4.2. Steps 2-3. Approximate radial symmetry and conclusion. We consider $n+1$ orthogonal directions $e_{1}, \ldots, e_{n+1}$, and we denote by $\pi_{1}, \ldots, \pi_{n+1}$ the corresponding critical hyperplanes. Let

$$
\mathcal{O}=\bigcap_{i=1}^{n+1} \pi_{i},
$$

and denote by $\mathcal{R}(p)$ the reflection of $p$ in $\mathcal{O}$. We have the following Lemma which extends Theorem 4.1 ,

Lemma 4.7. For any point $p \in S$ there exists a point $q \in S$ such that

$$
|\mathcal{R}(p)-q| \leq(n+1) C \operatorname{osc}(H) .
$$

Proof. We write $\mathcal{R}$ as

$$
\mathcal{R}=\mathcal{R}_{n+1} \circ \cdots \circ \mathcal{R}_{1}
$$

where $\mathcal{R}_{i}$ is the reflection about $\pi_{i}, i=1, \ldots, N+1$. By iterating Theorem $4.1 n+1$ times, we conclude.

As in [1, Proposition 6] we have that, for every direction $\omega$, it holds that

$$
\operatorname{dist}\left(\mathcal{O}, \pi_{m}\right) \leq C \operatorname{osc}(H),
$$

where $\pi_{m}$ is the critical hyperplane in the direction $\omega$ and $C$ is a constant that depends only on $\rho$ and $\operatorname{diam} S$, where

$$
\operatorname{diam} S=\max _{p, q \in S}|p-q| \text {. }
$$

We notice that diam $S$ can be bounded in terms of $|S|$ and $\rho^{-1}$. Indeed, let $p, q \in S$ be such that $|p-q|=\operatorname{diam} S$. By arguing as in the proof of Lemma 3.2, we can find a piecewise geodesic path on $S$ joining $p$ and $q$, and with length bounded by (39) (with $\delta=\rho / 2$ there), and then

$$
\operatorname{diam} S \leq \frac{|S| 2^{2 n}}{\omega_{n} \rho^{n}} .
$$

Hence, the constant $C$ in 84 can be bounded in terms of the dimension $n$ and upper bounds on $\rho^{-1}$ and $|S|$.

Finally, the bound on the difference of the radii (3) of the approximating balls is obtained by arguing as in [1, Proposition 7]. Indeed, we define

$$
r_{i}=\min _{p \in S}|p-\mathcal{O}|, \quad \text { and } \quad r_{e}=\max _{p \in S}|p-\mathcal{O}|,
$$

assume that the minimum and maximum are attained at $p_{i}$ and $p_{e}$, respectively, we obtain that

$$
r_{e}-r_{i} \leq 2 \operatorname{dist}(\mathcal{O}, \pi),
$$

where $\pi$ is the critical hyperplane in the direction

$$
\frac{p_{e}-p_{i}}{\left|p_{e}-p_{i}\right|}
$$

From (84) we conclude. 


\section{Proof of Corollary 1.2}

Lemma 5.1. Let $S$ be a closed $C^{2}$ hypersurface embedded in $\mathbb{R}^{n+1}$ and assume

$$
S \subset \bar{B}_{r_{e}} \backslash B_{r_{i}}
$$

with $r_{e}-r_{i} \leq 2 \rho$. Then

$$
\frac{p}{|p|} \cdot \nu_{p} \leq-1+\frac{1}{\rho}\left(r_{e}-r_{i}\right)
$$

for every $p \in S$.

Proof. Let $p \in S$ and let $c^{-}$and $c^{+}$be the centers of the interior and the exterior touching balls of radius $\rho$ tangent at $p$, respectively. Then

$$
\left|c^{-}+\frac{c^{-}}{\left|c^{-}\right|} \rho\right|=\sup _{q \in B_{\rho}\left(c^{-}\right)}|q| \leq r_{e}, \quad\left|c^{+}-\frac{c^{+}}{\left|c^{+}\right|} \rho\right|=\inf _{q \in B_{\rho}\left(c^{+}\right)}|q| \geq r_{i},
$$

and so

$$
\left|c^{-}+\frac{c^{-}}{\left|c^{-}\right|} \rho\right|^{2}-\left|c^{+}-\frac{c^{+}}{\left|c^{+}\right|} \rho\right|^{2} \leq r_{e}^{2}-r_{i}^{2}
$$

Therefore

$$
\left|c^{-}\right|^{2}+2 \rho\left|c^{-}\right|-\left|c^{+}\right|^{2}+2 \rho\left|c^{+}\right| \leq r_{e}^{2}-r_{i}^{2} .
$$

Taking into account that

$$
c^{+}=p-\rho \nu_{p}, \quad c^{-}=p+\rho \nu_{p}
$$

we get

$$
4 \rho p \cdot \nu_{p}+2 \rho\left(\left|c^{-}\right|+\left|c^{+}\right|\right) \leq r_{e}^{2}-r_{i}^{2}
$$

and so

$$
\frac{p}{|p|} \cdot \nu(p) \leq-\frac{\left|c^{-}\right|+\left|c^{+}\right|}{2|p|}+\frac{r_{e}+r_{i}}{4 \rho|p|}\left(r_{e}-r_{i}\right)
$$

Since

$$
\left|c^{-}\right|+\left|c^{+}\right| \geq\left|c^{-}+c^{+}\right|=2|\rho|
$$

and

$$
r_{e}=r_{i}+\left(r_{e}-r_{i}\right) \leq|p|+\left(r_{e}-r_{i}\right)
$$

we have that

$$
\frac{p}{|p|} \cdot \nu_{p} \leq-1+\frac{r_{e}-r_{i}}{2 \rho}+\frac{\left(r_{e}-r_{i}\right)^{2}}{4 \rho^{2}} \leq-1+\frac{r_{e}-r_{i}}{\rho}
$$

as required.

Now we are ready to prove Corollary 1.2 .

Proof. Step 1: $S$ is diffeomorphic to a sphere. In view of Theorem 1.1, there exists $\tilde{\varepsilon}$ and $C$ such that if $\operatorname{osc}(H)<\tilde{\varepsilon}$, then (2) and (3) hold. We may assume the concentric balls $B_{r_{e}}$ and $B_{r_{i}}$ centred in the origin. Let

$$
\varepsilon=\min \left\{\tilde{\varepsilon}, \frac{\rho}{2 C}\right\} .
$$

Hence the assumptions in Lemma 5.1 are satisfied. We consider the map $\varphi: S \rightarrow \partial B_{r_{i}}$, defined by

$$
\varphi(p)=r_{i} \frac{p}{|p|}
$$


We show that $\varphi$ a diffeomorphism. It is clear that $\varphi$ is smooth. Since $B_{r_{i}}$ is contained in the bounded domain enclosed by $S$, then $\varphi$ is surjective. Indeed, if $\zeta \in \partial B_{r_{i}}$, then

$$
\left\{\begin{array}{l}
\operatorname{dist}_{S}(\zeta) \leq 0 \\
\operatorname{dist}_{S}\left(\left(r_{e}-r_{i}\right) \zeta\right) \geq 0
\end{array}\right.
$$

and, by continuity, there exists a $t \geq 0$ such that $\operatorname{dist}_{S}((1+t) \zeta)=0$, i.e. $\zeta \in \varphi(S)$. Hence, assumption (1) plays a role only for proving the injectivity of $\varphi$. Let $p, q \in S$ and assume by contradiction that $\varphi(p)=\varphi(q)$. Then we may assume that $|p|<|q|$. Let $c^{+}=p-\rho \nu_{p}$ be the center of the exterior touching ball to $S$ at $p$. Since $p /|p|=q /|q|$, we have

$$
\left|q-c^{+}\right|^{2}=\left|(|q|-|p|) \frac{p}{|p|}+\rho \nu(p)\right|^{2}=(|q|-|p|)^{2}+\rho^{2}+2 \rho(|q|-|p|) \frac{p}{|p|} \cdot \nu_{p} .
$$

From Lemma 5.1 and since $|q|-|p| \leq r_{e}-r_{i}$, we have that

$$
\left|q-c^{+}\right|^{2} \leq\left(r_{e}-r_{i}\right)^{2}+\rho^{2}+2 \rho\left(r_{e}-r_{i}\right)\left(-1+\frac{r_{e}-r_{i}}{\rho}\right)=\rho^{2}-\left(r_{e}-r_{i}\right)\left(2 \rho-3\left(r_{e}-r_{i}\right)\right) .
$$

The choice of $\varepsilon$, as in 85 implies that $\left|q-c^{+}\right|<\rho$ which gives a contradiction.

Step 2: proof of (4). We denote by $F: \partial B_{r_{i}} \rightarrow S$ the inverse of the map $\varphi: S \rightarrow \partial B_{r_{i}}$ considered in the first step. We can write $F(\zeta)=\zeta+\Psi(\zeta) \frac{\zeta}{r_{i}}$ for every $\zeta$ in $\partial B_{r_{i}}$ and from Step

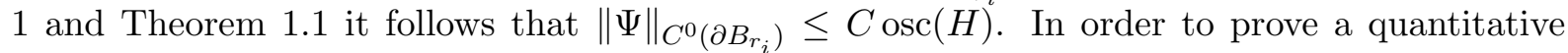
bound on the $C^{-}$-norm of the derivatives of $\Psi$, we work in the same fashion as in the proof of Lemma 3.4 .

Let $\zeta$ be a fixed point on $\partial B_{r_{i}}$ and set $p=F(\zeta)$ (i.e. $\zeta=r_{i} p /|p|$ ). Let $T_{\zeta}$ and $T_{p}$ be the tangent spaces to $\partial B_{r_{i}}$ at $\zeta$ and to $S$ at $p$, respectively. We can locally write $S$ around $p$ as

$$
q=p+x+u(x) \nu_{p}
$$

where $x$ belongs to a small neighborhood of the origin $O$ and $u$ is a $C^{2}$ map satisfying $u(O)=0$ and $\nabla u(O)=0$. Without loss of generality, we can assume that $\zeta=r_{i} e_{n+1}$ so that

$$
T_{\zeta}=\left\{x \in \mathbb{R}^{n+1}: x_{n+1}=0\right\},
$$

and we locally write $\partial B_{r_{i}}$ as $\zeta^{\prime}=\zeta+x+\eta(x) \nu_{\zeta}$, where $\eta(x)=r_{i}-\sqrt{r_{i}^{2}-|x|^{2}}$.

As in the proof of Lemma 3.4, we can chose an orthogonal matrix $A \in \mathrm{SO}(n+1)$ satisfying $A(\zeta)=-r_{i} \nu_{p}$ (we recall that $\left.\nu_{\zeta}=-\zeta / r_{i}\right)$, and we can locally write

$$
p+A x+u(A x) \nu_{p}=p+x+v(x) \nu_{\zeta}
$$

furthermore, $A$ is such that

$$
|A-I| \leq 2 \sqrt{1-\nu_{\zeta} \cdot \nu_{p}}
$$

We firstly prove that

$$
\partial_{x_{k}} \psi(O)=-\frac{1}{r_{i}} \partial_{x_{k}} v(O), \quad k=1, \ldots, n .
$$

Indeed, by setting $\psi=\Psi \circ \eta$, we have

$$
p+x+v(x) \nu_{\zeta}=\eta(x)-\psi(x) \nu_{\eta(x)},
$$

which implies

$$
p \cdot \nu_{\eta(x)}+x \cdot \nu_{\eta(x)}+v(x) \nu_{\zeta} \cdot \nu_{\eta(x)}-\eta(x) \cdot \nu_{\eta(x)}=-\psi(x),
$$


i.e.

$$
\frac{1}{r_{i}} p \cdot \eta(x)+\frac{1}{r_{i}} x \cdot \eta(x)+\frac{1}{r_{i}} v(x) \nu_{\zeta} \cdot \eta(x)-r_{i}=\psi(x),
$$

where we have used that $\nu_{\eta(x)}=-\eta(x) / r_{i}$. From $\eta(O)=\zeta$ and $v(O)=0$ we obtain (88).

Now, we give a bound on the derivatives of $v$ at $O$ and in terms of the difference $r_{e}-r_{i}$. We notice that 86 implies

$$
v(x)=(A-I) x \cdot \nu_{\zeta}+u(A x) \nu_{p} \cdot \nu_{\zeta},
$$

and, since $|\nabla u(O)|=0$, we obtain that

$$
\left|\partial_{x_{k}} v(O)\right| \leq|A-I|, \quad k=1, \ldots, n .
$$

From (87) and Lemma 5.1 we obtain that

$$
\left|\partial_{x_{k}} v(O)\right| \leq 2 \sqrt{\frac{r_{e}-r_{i}}{\rho}}, \quad k=1, \ldots, n,
$$

and from (3) and (88) we find (4) and we conclude.

Remark 5.2. As emphasized in the Introduction, if we assume that $\rho$ is not bounded from below, it is possible to construct a family of closed surfaces embedded in $\mathbb{R}^{3}$, not diffeomorphic to a sphere, with $\operatorname{osc}(H)$ arbitrarly small and such that (3) fails. For instance one can consider the following example, suggested us by A. Ros, done by gluing almost pieces of unduloids.

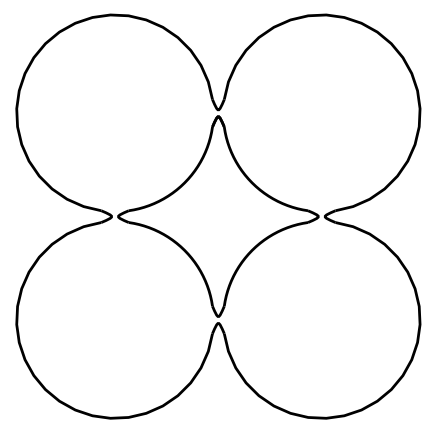

\section{REFERENCES}

[1] A. Aftalion, J. Busca, W. Reichel, Approximate radial symmetry for overdetermined boundary value problems, Adv. Diff. Eq. 4 no. 6 (1999), 907-932.

[2] A. D. Alexandrov, Uniqueness theorems for surfaces in the large II, Vestnik Leningrad Univ. 12, no. 7 (1957), 15-44. (English translation: Amer. Math. Soc. Translations, Ser. 2, 21 (1962), 354-388.)

[3] A. D. Alexandrov, Uniqueness theorems for surfaces in the large V, Vestnik Leningrad Univ. 13, no. 19 (1958), 5-8. (English translation: Amer. Math. Soc. Translations, Ser. 2, 21 (1962), 412-415.)

[4] A. D. Alexandrov, A characteristic property of spheres, Ann. Mat. Pura Appl., 58 (1962), 303-315.

[5] W. K. Allard, On the first variation of a varifold, Ann. Math., 95 (1972), 417-491.

[6] F. J. JR. Almgren, Plateaus problem. An invitation to varifold geometry, Mathematics Monograph Series, W. A. Benjamin, Inc., New York-Amsterdam, 1966.

[7] R. Arnold, On the Alexandrov-Fenchel Inequality and the Stability of the Sphere, Monatsh. Math., 155 (1993), 1-11.

[8] H. Berestycki, L. A. Caffarelli, L. Nirenberg, Inequalities for second-order elliptic equations with applications to unbounded domains I, Duke Math. J., 81 (1996), no. 2, 467-494.

[9] B. Brandolini, C. Nitsch, P. Salani, C. Trombetti, On the stability of the Serrin problem, J. Diff. Equations 245 (2008), 1566-1583. 
[10] S. Brendle, Constant mean curvature surfaces in warped product manifolds, Publ. Math. Inst. Hautes Études Sci. 117 (2013), 247-269.

[11] S. Brendle, M. Eichmair, Isoperimetric and Weingarten surfaces in the Schwarzschild manifold, J. Diff. Geom., 94 (2013), 387-407.

[12] X. Cabré, M. Fall, J. Sola-Morales, T. Weth, Curves and surfaces with constant nonlocal mean curvature: meeting Alexandrov and Delaunay. Preprint, 2015. arXiv:1503.00469.

[13] L. Caffarelli, B. Gidas, J. Spruck, Asymptotic symmetry and local behavior of semilinear elliptic equations with critical Sobolev growth. Comm. Pure Appl. Math., 42 (1989), 271-297.

[14] L. Caffarelli, S. SAlsa, A geometric approach to free boundary problems. Graduate Studies in Mathematics, 68. American Mathematical Society, Providence, RI, 2005.

[15] D. Christodoulou, S. T. Yau, Some remarks on the quasi-local mass, Contemp. Math. 71 (1988), 9-14.

[16] G. Ciraolo, A. Figalli, F. Maggi, M. Novaga, Rigidity and sharp stability estimates for hypersurfaces with constant and almost-constant nonlocal mean curvature. To appear in J. Reine Angew. Math. (Crelle's Journal). arXiv: 1503.00653.

[17] G. Ciraolo, F. Maggi, On the shape of compact hypersurfaces with almost constant mean curvature. Preprint. arXiv: 1503.06674.

[18] G. Ciraolo, R. Magnanini, S. Sakaguchi, Symmetry of solutions of elliptic and parabolic equations with a level surface parallel to the boundary, J. Eur. Math. Soc. (JEMS) 17 (2015), 2789-2804.

[19] G. Ciraolo, R. Magnanini, S. Sakaguchi, Solutions of elliptic equations with a level surface parallel to the boundary: stability of the radial configuration, to appear in J. Analyse Math. arXiv:1307.1257.

[20] G. Ciraolo, R. Magnanini, V. Vespri, Hölder stability for Serrin's overdetermined problem. Preprint. arXiv: 1410.7791.

[21] G. Ciraolo, R. Magnanini, V. Vespri, Symmetry and linear stability in Serrin's overdetermined problem via the stability of the parallel surface problem. Preprint.

[22] C. De Lellis, S. Müller, Optimal rigidity estimates for nearly umbilical surfaces. J. Differential Geom. 69 (2005), no. 1, 75-110.

[23] C. De Lellis, S. Müller, A $C^{0}$ estimate for nearly umbilical surfaces. Calc. Var. Partial Differential Equations 26 (2006), no. 3, 283-296.

[24] M. P. Do Carmo, H. B. Lawson, On the Alexandrov-Bernstein Theorems in Hyperbolic Space, Duke Math Journal 50 (1983), 995-1003.

[25] B. Gidas, W. M. Ni, L. Nirenberg, Symmetry and related properties via the maximum principle, Comm. Math. Phys., 68 (1979), 209-243.

[26] D. Gilbarg, N. S. Trudinger, Elliptic partial differential equations of second order, Springer-Verlag, BerlinNew York, 1977.

[27] M. Gromov, Stability and pinching. Geometry Seminars. Sessions on Topology and Geometry of Manifolds, (Bologna, 1990), Univ. Stud. Bologna, Bologna, 1992, 55-97.

[28] W.-Y. Hsiang, Z.-H. Teng, W.-C. YU, New examples of constant mean curvature immersions of $(2 k-1)-$ spheres into Euclidean 2k-space, Ann. of Math. (2) 117 (1983), 609-625.

[29] G. Huisken, S.-T. YAu, Definition of center of mass for isolated physical systems and unique foliations by stable spheres with constant mean curvature, Invent. Math. 124 (1996), no. 1-3, 281-311.

[30] N. J. KorevaAR, Sphere theorems via Alexsandrov for constant Weingarten curvature hypersurfaces - Appendix to a note of A. Ros, J. Diff. Geom., 27 (1988), 221-223.

[31] P. Kohlmann, Curvature measures and stability, J. Geom., 68 (2000), 142-154.

[32] D. Koutroufiotis, Ovaloids which are almost Spheres, Comm. Pure Appl. Math., 24 (1971), 289-300.

[33] N. J. Korevaar, R. Kusner, B. Solomon, The structure of complete embedded surfaces with constant mean curvature, J. Diff. Geom., 30 (1989), 465-503.

[34] N. J. Korevaar, R. Mazzeo, F. Pacard, R. Schoen. Refined asymptotics for constant scalar curvature metrics with isolated singularities, Invent.Math., 135 (1999), 233-272 .

[35] U. Lang, Diameter Bounds for Convex Surfaces with Pinched Mean Curvature, Manuscripta Math., 86 (1995), 15-22.

[36] C. LI, Mononicity and symmetry of solutions of fully nonlinear elliptic equations on bounded domains, Commun. Part. Diff. Eq., 16 (1991), 491-526.

[37] C. LI, Mononicity and symmetry of solutions of fully nonlinear elliptic equations on unbounded domains, Commun. Part. Diff. Eq., 16 (1991), 585-615.

[38] W. MeEks III, The topology and geometry of embedded surfaces of constant mean curvature, J. Diff. Geom. 27 (1988), 539-552. 
[39] S. Montiel, Unicity of constant mean curvature hypersurfaces in some Riemannian manifolds, Indiana Univ. Math. J., 48 (1999), 711-748.

[40] J. D. Moore, Almost Spherical Convex Hypersurfaces, Trans. Amer. Math. Soc., 180 (1973), $347-358$.

[41] S. Montiel, A. Ros, Compact hypersurfaces: The Alexandrov theorem for higher order mean curvatures, Pitman Monographs and Surveys in Pure and Applied Mathematics 52 (1991), 279-296.

[42] R. ReILly, Applications of the Hessian operator in a Riemannian manifold, Indiana Univ. Math. J., 26 (1977), 459-472.

[43] A. Ros, Compact hypersurfaces with constant scalar curvature and a congruence theorem, J. Diff. Geom., 27 (1988), 215-220.

[44] A. Ros, Compact hypersurfaces with constant higher order mean curvatures, Rev. Math. Iber., 3 (1987), 447-453.

[45] J. Serrin, A symmetry problem in potential theory, Arch. Rational Mech. Anal. 43 (1971), 304-318.

[46] R. Schneider, A Stability Estimate for the Alexandrov-Fenchel Inequality, with an Application to Mean Curvature, Manuscripta Math., 69 (1990), 291-300.

[47] R. Schoen, Uniqueness, symmetry, and embeddedness of minimal surfaces, J. Diff. Geom., 18 (1983), 791809.

[48] H. C. Wente, Counterexample to a conjecture of H. Hopf, Pacific J. Math. 121 (1986), $193-243$.

[49] S.-T. YAu, Submanifolds with constant mean curvature I, Amer. J. Math. 96 (1974), 346-366

Dipartimento di Matematica e Informatica, Università di Palermo, Via Archirafi 34, 90123, Italy

E-mail address: giulio.ciraolo@unipa.it

Dipartimento di Matematica G. Peano, Università di Torino, Via Carlo Alberto 10, 10123 TORINO, ITALY.

E-mail address: luigi.vezzoni@unito.it 